\title{
Improved damage detectability in a wind turbine blade by optimal selection of vibration signal correlation coefficients
}

\section{Simon Hoell and Piotr Omenzetter}

\begin{abstract}
The central message of this paper is that for robust and efficient damage detection the damage sensitive features (DSFs) should be selected optimally in a systematic way such that only these features that contribute the most to damage detectability be retained. Furthermore, suitable transformations of the original features may also enhance damage detectability. We explore these principles using data from a wind turbine blade. Several damage extent scenarios are introduced non-destructively. Partial autocorrelation coefficients (PACCs) are proposed as vibrationbased DSFs. Scores calculated with principal component analysis (PCA) of PACCs are the transformed DSFs. Statistical distances between the DSF subsets estimated from the healthy and a reference damage state are calculated with respect to a statistical threshold as a measure of optimality. The fast forward (FF) method and a genetic algorithm are used to optimize the detectability of damage by DSF selection. The comparison between the two methods points out that FF offers a comparable performance at a lower computational cost. The classifiers based on the optimal features are tested on data from several previously unseen healthy and damaged cases and across a range of statistical detection thresholds. It is demonstrated that the selected PCA scores of the PACCs are superior compared to the initial features and allow identifying small damage confidently.
\end{abstract}

\section{Keywords}

Damage detection, fast forward selection, genetic algorithm, optimal feature selection, partial autocorrelation function, principal component analysis, structural health monitoring, vibration based damage detection, wind turbines

\section{List of abbreviations}

AUC Area under curve

DSF Damage sensitive feature

FC Fisher criterion

FF Fast forward

GA Genetic algorithm

PACC Partial autocorrelation coefficient

PACF Partial autocorrelation function

PC Principal component

PCA Principal component analysis

ROC Receiver operating characteristic

SDD Structural damage detection

SHM Structural health monitoring

WTB Wind turbine blade

\section{List of symbols}

\section{Roman letters}

$D \quad$ Mahalanobis distance

$F \quad$ Cumulative probability distribution function

$H \quad$ Hypothesis

$\mathcal{N} \quad$ Gaussian probability distribution

$\mathcal{P} \quad$ Parental population for genetic algorithm

$S \quad$ Vector cosine distance

S Score matrix

$T^{2} \quad$ Hotelling's $T^{2}$ statistic

T Principal component matrix

V Damage sensitive feature matrix

a Autoregressive coefficient

$b \quad$ Binary selection indicator

b Binary selection vector

$f \quad$ Fitness function

$m$ Dimensionality of damage sensitive feature vector $n \quad$ Number of samples; Index

$p \quad$ Autoregressive order

$r \quad$ Autocorrelation function

$s \quad$ Principal component score

s Principal component score vector

$t \quad$ Discrete time

t Principal component

$v \quad$ Damage sensitive feature

v Damage sensitive feature vector

$x \quad$ Time series

$z \quad$ Zero-mean, unit-variance normally distributed time series

\section{Greek letters}

$\begin{array}{ll}\Delta & \text { Difference operator } \\ \Lambda & \text { Eigenvalue matrix } \\ \boldsymbol{\Sigma} & \text { Variance-covariance matrix }\end{array}$

The LRF Centre for Safety and Reliability Engineering, The University of Aberdeen, Aberdeen, UK

\section{Corresponding author:}

Piotr Omenzetter, The LRF Centre for Safety and Reliability Engineering, The University of Aberdeen, Aberdeen AB24 3UE, UK.

Email: piotr.omenzetter@abdn.ac.uk 


$\begin{array}{ll}\mathcal{X}^{2} & \text { Chi-square probability distribution function } \\ \alpha & \text { Level of significance; Partial autocorrelation } \\ & \text { function } \\ \beta & \text { Mutation rate } \\ \gamma & \text { Number of offsprings } \\ \kappa & \text { Population size } \\ \lambda & \text { Eigenvalue } \\ \mu & \text { Mean value } \\ \mu & \text { Mean value vector } \\ \rho & \text { Correlation coefficient } \\ \sigma & \text { Standard deviation } \\ \sigma^{2} & \text { Variance } \\ \tau & \text { Time lag }\end{array}$

\section{Subscripts}

$0 \quad$ Null hypothesis

1 Alternative hypothesis

$c \quad$ Current state

crit Critical

$d \quad$ Damage state

$h \quad$ Healthy state

opt Optimal

pl Pooled

rel Relative

samp Sample

sol Solution

\section{Superscripts}

T Transpose

\section{Diacritics}

$\begin{array}{ll}\hat{i} & \text { Estimate } \\ , & \text { Normalized } \\ \prime \prime & \text { Tempring } \\ & \end{array}$

\author{
Operators \\ Corr Correlation \\ min Minimum value \\ (...) $\mid(\ldots)$ Conditional estimate \\ $|(\ldots)| \quad$ Absolute value
}

\section{Introduction}

The relentless demands of economic competitiveness in the environment of increasing public interest in enhanced environmental friendliness, reliability and safety of operations of energy, aerospace, mechanical, civil, structural and other man-made systems create favourable conditions for advancing the developments and use of intelligent systems. Those systems are characterized by the ability to infer their own states and these of their surroundings and control their responses and properties on the basis of this knowledge under varying environmental and operational conditions to guarantee optimal operation. Structural health monitoring (SHM) can be defined as the process of determining the current structural condition with the help of knowledge and information extracted from measurements (Farrar and Worden, 2007), and therefore is an important building block of intelligent systems. SHM technologies based on different physical principles allow observing various quantities to describe the state of a structure. Technologies based on ultrasonic and elastic wave generation and detection use generally active systems with artificial excitation (Gopalakrishnan et al., 2011; TorresArredondo et al., 2015; Liu and Chattopadhyay, 2013), which add to their complexity. This can be avoided by passive sensing approaches where ambient excitations from environmental sources, such as wind, earthquakes and waves, from the operations, for example in the case of bridges with traffic or rotating machinery, or developing damage itself are sufficient. Acoustic emissions and strains can be measured with such systems (Farhidzadeh et al., 2013; Meo, 2014; Benedetti et al., 2011; Li, 2010; Ciang et al., 2008), but dense sensor arrays are required to identify local damage. This adversely affects instrumentation and data analysis costs. Utilizing global structural vibrations resulting from ambient excitations can be more efficient because the response encompasses the entire structure due to low damping and long wave lengths. Since vibrational responses are affected by changes in stiffness, mass or energy dissipation of a structure, which are altered by damage, they are suitable for SHM applications.

In the past, the development of vibration-based SHM techniques attracted considerable attention (Doebling et al., 1998, 1996; Fan and Qiao, 2011; Brownjohn et al., 2011; Sohn et al., 2004). This led to definition of different damage sensitive features (DSFs) for describing the current structural state. The majority of these DSFs are multivariate and can be used to construct DSF vectors of different dimensionalities. Structural states appear then as specific patterns amongst the DSF vectors. Thus, decisions about the structural state can be made by comparing current DSF vectors with preassigned patterns following a statistical pattern recognition paradigm (Farrar et al., 2001). Here, implicitly learned or explicitly derived DSF distributions from different states enable to exclude or confirm structural states. Soft computing techniques, such as Gaussian mixture models (Chakraborty et al., 2014), self-organizing maps (Torres-Arredondo et al., 2013), fuzzy pattern recognition (da Silva et al., 2008), decision tree classifier (Kostka et al., 2013), learning vector quantization (de Lautour and Omenzetter, 2010b), artificial neural networks (Bandara et al., 2014a), artificial immune pattern recognition (Chen and Zang, 2011) and support vector machines (Farrar et al., 2001), can be used for pattern recognition. On the other hand, the conceptual clarity and the possibility of defining damage detection thresholds systematically make approaches utilizing statistical tests very attractive. Statistical hypothesis testing and statistical process control have been successfully applied in the context of vibration-based SHM applications in the past (Döhler et al., 2013; Sohn et al., 2000; Rizos et al., 2008; Kullaa, 2003; Wang and Ong, 2009). Fassois and Sakellariou (2007) presented a unified statistical framework for time seriesbased structural damage detection (SDD).

Traditionally, modal parameters, i.e. natural frequencies, modal damping ratios, and mode shapes and their spatial derivatives, are used for the definition of DSFs (Carden and Fanning, 2004). For large structural systems, where forced vibration testing is impractical, operational modal analysis allows estimating these parameters (Reynders, 2012), often with the help of additional processing steps, 
e.g. hierarchical and fuzzy clustering (Reynders et al., 2012; Carden and Brownjohn, 2008). However, fully automatic identification of modal DSFs entails high computational demands and significant practical challenges, especially in noisy conditions typical of real life applications, that limit the applicability of these approaches.

Parametric and non-parametric time series representations of vibration response signals are far less demanding. Autoregressive and mixed autoregressive moving average models are examples of parametric models. The relationship between autoregressive coefficients and structural stiffness has been theoretically investigated by Nair et al. (2006). The parametric models enable to define DSFs in terms of the estimated model coefficients or on the basis of residuals calculated from a baseline model and the recorded current time series. Their performance with respect to the detectability of damages depends on the selection of an appropriate model type and order (Figueiredo et al., 2011). Nair et al. (2006) proposed an index based on the first three autoregressive coefficients and used statistical testing of the index mean for damage detection. A more systematic selection of autoregressive coefficients was performed by de Lautour and Omenzetter (2010a), where the trends between increasing the numbers of sensors and autoregressive coefficients with respect to damage classification errors were explored. In that study, larger autoregressive coefficient sets improved the results of damage classification by means of an artificial neural network. Approaches based on model residuals generally require the use of the full autoregressive coefficient sets (Lu et al., 2008). Nevertheless, the selection of a model structure with an appropriate model order, identification of model coefficients and validation of the estimated model is necessary for parametric time series representations (Box et al., 2008) adding to the amount of effort required for creating a successful damage detection tool.

In contrast, non-parametric time series representations are simple and computationally efficient to obtain, albeit they are less parsimonious. They can be defined in the frequency, dual time-frequency or time domain. Common frequency domain representations for SHM are power spectral densities (Kumar et al., 2012), transfer functions (Ghoshal et al., 2000) and frequency response functions (Manson and Barthorpe, 2010; Papatheou et al., 2010). Using frequency domain representations, pre-defined narrow frequency bands or even individual peaks are used to improve damage detectability by focusing on features most affected by damage. Empirical mode decomposition and wavelet transformation are dual time-frequency representations. They are particularly useful in non-stationary and/or non-linear cases. The applicability of empirical mode decomposition for SHM in a cantilever steel beam under impulse excitation for non-linear system identification is studied by Chen et al. (2014), where it is shown that distance measures between instantaneous mode shapes allow detecting and localizing vibro-impact damage. While using wavelet transformation for vibrationbased SHM, wavelet energies and entropies are often used to define DSFs (Nair and Kiremidjian, 2012; Lee et al., 2014), but wavelet transformation coefficients and scales need to be selected in advance.
Non-parametric time domain representations of vibration signals enable to define various DSF vectors, for example in terms of correlations between time series. The theoretical relationship between cross-correlations and frequency response functions is utilized by Yang et al. (2007). They used cross-correlation amplitudes from different sensors for SDD in numerical and experimental beams. Crosscorrelations at the zero lag are used for SDD in a numerical composite laminate beam (Yang et al., 2009). Zhang and Schmidt (2014) demonstrated that autocorrelations at the zero lag enable better detectability of damage in a twelve storey shear frame structure under white noise excitation compared to using cross-correlations. Maximum cross-correlation coefficients are successfully applied for delamination assessment in small composite beams under Gaussian excitation (Trendafilova et al., 2015). Green's functions, as time derivatives of cross-correlations, are used for SDD in a small-scale wind turbine blade (WTB) (Tippmann et al., 2015), where the performance of different statistics and metrics are investigated including signal energies for selected frequency ranges.

In SHM, feature extraction by means of principal component analysis (PCA) received considerable attention, and deserves a review as this technique is also used in our present study. Removal of operational and environmental effects, dimensionality reduction and extraction of transformed DSFs with higher sensitivity to damage are the main purposes. The property of PCA to identify a projection defined by orthogonal basis vectors which maximizes the variation in the dataset for the first few dimensions is an attractive property and is often utilized (Jolliffe, 2002). For the removal of adverse effects, it is generally assumed that the highest variations in a dataset are the result of these influences, thus rejecting the first few principal components (PCs) can counteract the issues. Yan et al. (2005) used this approach for SDD in numerical and experimental bridge studies to remove temperature effects. A comparison between PCA, manual selection with respect to univariate novelty measures and cointegration demonstrated the performance of PCA for SDD with Lamb waves in a composite plate with temperature variation (Cross et al., 2012). Nowadays, lower costs of sensing technology allow observing structures with high resolution in time and space. Higher dimensional feature spaces are one result, which can significantly affect the detectability of damages due to the 'curse of dimensionality' (Zimek et al., 2012). The damage sensitivity of control charts established for vibration-based DSFs is increased by reducing the initial feature dimensionality with the help of PCA (Kullaa, 2003). Frequency response function based DSFs are reduced by PCA in order to apply artificial neural networks for prediction of damage location and severity in a numerical shear-frame structure (Bandara et al., 2014b). Mohd Aris et al. (2014) used the first two PCs to assess the effects of damage and restoration in a carbon fibre reinforced composite plate interrogated via wavelet transforms of Lamb wave signals. The use of DSFs transformed through PCA enabled to distinguish between different states. PCA scores, as transformed DSFs, can be calculated by projecting initial DSFs by PCs onto a new feature space of equal or lower dimension depending on the actual PC selection. Mujica et al. (2014) proposed the use 
of univariate statistical tests of scores for making decisions about the structural state. They applied the approach to mechanical response data obtained from piezoelectric transducers attached to a small aluminium plate under Lamb wave excitation. More advanced techniques, such as non-linear PCA (Torres-Arredondo et al., 2015), kernel PCA (Liu and Chattopadhyay, 2013) and independent component analysis (Hernandez-Garcia and Masri, 2014), allow accounting for non-linear relations between DSFs and have also received attention.

However, the main premise of this study is that no mater what DSFs are employed, adopting only these features, possibly after an additional transformation, that allow optimal damage detectability is critical for the success of any SDD algorithm. Judicious feature selection can boost damage detectability significantly, and, equally importantly, failing to eliminate features which are noisy and/or of poor sensitivity to damage can easily blunt the algorithm power. However, this seemingly natural feature subset selection is mainly done in SHM studies in unstructured ways, often ad hoc and by trial and error without systematic investigation. This is inadequate in settings with high dimensional DSF vectors, small sample numbers for estimating their distributions as description of patterns, and restrictions imposed on computational effort.

For statistical pattern recognition in general, different techniques are already developed for feature selection (Jain et al., 2000), while only a limited number of their applications can be found in SHM studies. Fast forward (FF) selection is a simple and computationally efficient method, where features are consecutively added to a multivariate DSF vector in order to maximize an objective function. Park et al. (2010) applied FF selection for data reduction to facilitate efficient data transmission in wireless sensor networks. They selected wavelet transform coefficients from Lamb wave signals for transmission with respect to the damage detection rate for a training dataset. Sequential forward selection is applied by Zugasti et al. (2013) to select acceleration signals for SDD in a laboratory tower structure with respect to a mutual information criterion using healthy state measurements only. Furthermore, the effect of PCA as feature extraction method is studied, where only PCs with the highest contribution to the initial variance were retained. It was found that while feature extraction and selection used separately decreased the damage sensitivity, a combination of both approaches led to improved outcomes. A genetic algorithm (GA) was applied for enhanced selection of transmissibility function ranges by Worden et al. (2008), to improve initial manual selections. The approach was used to optimize an artificial neural network based damage location classifier for an aircraft wing structure. Adaptive boosting is another approach for selecting features. It iteratively modifies simple base classifiers to improve the overall error rate. Ying et al. (2013) used this technique for SDD in an experimental pipe with ultrasonic measurements. Feature assimilation is a similar idea. Here, different metrics or statistics of weak DSFs are summed to improve damage detectability. Prabhu and Atamturktur (2013) calculated sums of different vibration-based DSFs for experimental SDD in a small plastic arch model.
However, the reviewed approaches highlight the need for a systematic methodology for transforming and selecting multivariate DSFs for improving damage detectability. Many existing studies often assert, rather than clearly demonstrate or prove, that transformed DSFs will perform better without thorough evaluation and comparison of the initial feature performance. Neither do they explicitly evaluate the interplay between the dimensionality of the initial or transformed DSF vectors and statistical detection thresholds, and how that affects the detectability of early damages. Motivated by these limitations, the present paper addresses the issue of multivariate feature transformation and selection to improve the detectability of early damages. At first, DSFs of lower dimensionalities are directly selected from the original feature vectors. These features later compete with transformed DSFs obtained by PCA and then pruned by keeping only selected PCA scores, in a race to detect damage as early as possible. In both cases, the selection of features is based on the fact that adding another univariate DSF to a DSF vector only improves damage detectability if its contribution to the statistical distance between the healthy and damage DSF distributions is larger than the corresponding increase in the statistical threshold due to the increased dimensionality, i.e. the number of statistical degrees of freedom. Thus, there will be an optimal dimension of DSF vector that corresponds to the maximum ratio of the statistical distance to the threshold. It should be noted that our objectives stretch beyond merely reducing the size of a DSF, which often accepts some decline in damage detection performance. Feature reduction is here but a positive side effect of the far more beneficial optimal DSF selection. The aforementioned FF and GA-based selection strategies are explored for the identification of optimal DSF vectors, where decisions about the structural state are made by means of statistical hypothesis testing of statistical distances between the feature vectors of the healthy and a selected reference damage state. For the FF feature selection, the DSF dimension is increased one-by-one and the feature contributing the most to the statistical distance is retained. The second approach utilizes a binary GA as an evolutionary global optimization technique (Beyer and Schwefel, 2002) for the selection of the optimal DSF dimension. Here, dimensions are determined via random initialization of binary selection vectors, which are subsequently optimized by recombination, mutation and selection with respect to damage detectability.

The proposed methodology is applied to partial autocorrelation coefficients (PACCs) as initial DSFs. They are nonparametric representations of time series and are in this study applied to structural acceleration signals. PACCs can be estimated from autocorrelations by recursion or directly from the coefficients of autoregressive models of different orders. This illustrates the close relationship between these time series representations (Kay, 1988). Here, they are estimated for the data of laboratory experiments with a small-scale WTB made of glass-fibre reinforced epoxy composite and excited with the help of a domestic pedestal fan generating windlike excitation. Damage is introduced non-destructively by adding small masses at the WTB's trailing edge, where increasing masses simulate different damage extents. This 
approach, used not infrequently in laboratory damage detection studies (see e.g. Papatheou et al. (2010)), was adopted so as to preserve the intact blade for future experimental work. (While we refer to the added mass as 'damage', structural damage in real WTBs would rather take the form of stiffness reduction (due to delamination, disbonding, cracks, etc.) than mass change. Hence in our experiments we rather detect structural 'change or 'modification' than damage, but there is no reason to believe the presented methodology would perform substantially differently for stiffness losses.) While the proposed techniques can be extended to a wide range of structural systems, aside from providing a convenient laboratory model for exploring our SDD approach, a WTB is selected for experimental work because it is believed that SHM applications to wind turbines hold relatively more promise of bringing significant benefits compared to other types of structures. This is because of the accelerated interest in renewable wind energy world-wide leading to ever growing sizes of wind turbines and erections in remote areas, such as offshore where winds are stronger and more reliable. However, increasing operation and maintenance expenditure adversely affects the energy production targets and expected revenues because they can make up to $20 \%$ of the total energy production costs (Blanco, 2009). Designing and building wind turbines as smart structures with efficient SDD capabilities can help to mitigate this undesirable situation.

The paper is structured as follows. The first section presents the theoretical background with analytical and numerical tools of the proposed methodology. The advantages of an appropriate composition of DSFs are then demonstrated analytically for a bivariate DSF vector. The following section describes the experimental programme conducted on the small scale WTB. Next, the systematic optimal DSF selections by the different procedures considered are studied for the initial PACC-based DSF vectors and scores obtained by PCA transformation for the WTB. Additionally, the detection rates for increasing damage extents for different optimal DSF selections are presented to validate the method. Finally, a summary and conclusions round up the paper.

\section{Methodology and tools}

The proposed methodology for improved damage detectability by using optimally selected DSFs is illustrated with a flowchart in Figure 1. The methodology is general and can use different signals, DSFs and analytical tools. This paragraph explains the main general methodological steps highlighted in the flowchart and indicates our particular choices of signals to measure, DSFs and numerical processing algorithms; the latter appear in the flowchart written in a smaller sized font and in parentheses in the explanations to follow. The process starts with an offline baseline or training phase, where dynamic response signals (accelerations) from the healthy and a reference damage state are acquired and pre-processed (divided into segments and normalized). Then, the types of DSFs to be used are selected (PACCs or PCA scores of PACCs). DSFs are then extracted from the measured dynamic data in both structural states and the parameters of their statistical distributions estimated. Next, optimal DSF selections are identified (with the help of the FF or GA selection algorithms). The subsequent detection phase can then work online and make damage decisions by statistical hypothesis testing of optimally selected DSFs estimated from the current structural state at a chosen significance level.

It should be noted that the number of decisions to be made by the analyst is relatively small facilitating automation of the proposed damage detection method. The type and location of physical damage to be focused on should be predetermined for efficient operations taking into account its prevalence and risk it poses to structural integrity. Then, the sensor type (e.g. accelerometers or strain gauges), their sampling rates and locations have to be chosen, though the latter can also be a subject of numerical optimisation. Next, the specific type of DSF to be used has to be decided on, but the significant PACCs we employ can be computed, selected and transformed automatically with a minimum need for human judgement. The level of significance for for statistical hypothesis testing needs to be selected, but this level corresponds to the acceptable false positive and negative rates. The decisions outlined above are typical for most of the available damage detection schemes. To round up the training phase, an automatic selection of optimal subsets of DSFs is conducted. The subsequent damage detection phase does not require any additional parameters to be set. It simply compares automatically a statistic of the DSF extracted according to the approach established in the training phase to a threshold also decided during training.

The reminder of this section provides an overview of the methodological steps of the proposed approach to optimal DSF selection via explanations of the main conceptual, analytical and computational approaches and methods. The presentation is structured in such a way that the general ideas and concepts are laid out first moving on to more specific techniques and tools actually applied in the considered experimental case. Thus, statistical hypothesis testing is covered first as the overall framework for damage detection using a generic multivariate DSF. From there, the objective function for optimal DSF selection is formulated for robust damage decisions. As we propose to transform original DSFs by PCA to further enhance damage detectability, PCA is also briefly presented. The techniques to solve the optimality problem, FF and GA, are then detailed. The concept of receiver operational characteristic (ROC) is explained because we used it for assessing the performance of out methodology. Finally, partial autocorrelation function (PACF) is explained as it provides us with the actual features we utilize in this study.

\section{Statistical hypothesis testing and objective function for optimal feature selection}

The proposed methodology aspires to improve SDD decision making by differentiating between the healthy state and unknown damage states with the help of multivariate DSFs. A statistical model can be postulated for the inherent variations in the healthy state DSF estimates. A generic $m$ dimensional DSF vector, $\mathbf{v}_{i}$, extracted from the time series segment $i$ can be represented as:

$$
\mathbf{v}_{i}=\left[\begin{array}{llll}
v_{i 1} & v_{i 2} & \cdots & v_{i m}
\end{array}\right]^{T} \sim \mathcal{N}(\boldsymbol{\mu}, \boldsymbol{\Sigma})
$$




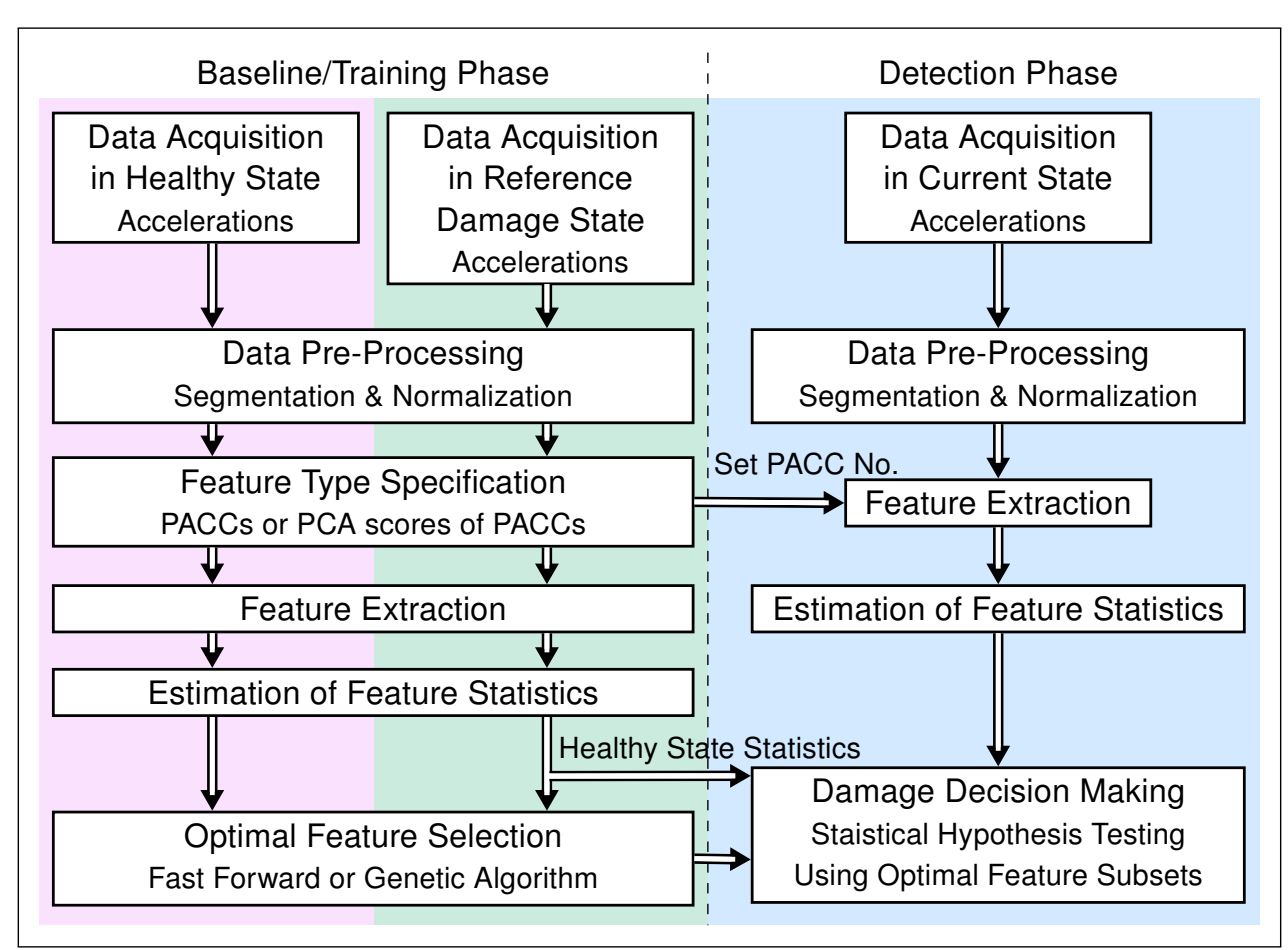

Figure 1. Process of optimal DSF selection and statistical damage decision making.

where ${ }^{T}$ denotes transpose and the multivariate Gaussian probability distribution, $\mathcal{N}(\boldsymbol{\mu}, \boldsymbol{\Sigma})$, is described by the true multivariate mean and the true variance-covariance matrix, $\boldsymbol{\mu}$ and $\boldsymbol{\Sigma}$, respectively.

To decide whether a structure still remains healthy or, conversely, damage has occurred, statistical hypothesis testing can be utilized. This enables to reach such decisions in a rigorous and quantitative way. Assuming that damage leads to a shift in the mean of the DSF vector, $\mathbf{v}$, the following statistical hypotheses compete:

$$
\begin{array}{lll}
H_{0}: & \boldsymbol{\mu}_{c}=\boldsymbol{\mu}_{h} & \text { (healthy) } \\
H_{1}: & \boldsymbol{\mu}_{c} \neq \boldsymbol{\mu}_{h} & \text { (damaged) }
\end{array}
$$

The healthy state corresponds to equality of the multivariate means from the current state, $\boldsymbol{\mu}_{c}$, and the reference healthy state, $\boldsymbol{\mu}_{h}$. This represents the null hypothesis, $H_{0}$. The competing alternative hypothesis, $H_{1}$, refers to the damage state. In practical applications, statistical models can usually only be constructed from limited numbers of samples, thus the true statistical properties are not available and can only be estimated. However, the $T^{2}(m)$ statistic defined as (Rencher, 1998):

$$
T^{2}(m)=\frac{n_{c} n_{h}}{n_{c}+n_{h}}\left(\hat{\boldsymbol{\mu}}_{c}-\hat{\boldsymbol{\mu}}_{h}\right)^{T} \hat{\boldsymbol{\Sigma}}_{p l}^{-1}\left(\hat{\boldsymbol{\mu}}_{c}-\hat{\boldsymbol{\mu}}_{h}\right)
$$

with $m$ being as previously the DSF vector dimensionality, can be used for testing the above hypothesis. The $T^{2}(m)$ statistic is the distance between two $m$ dimensional sample mean estimates, $\hat{\boldsymbol{\mu}}_{c}$ and $\hat{\boldsymbol{\mu}}_{h}$, standardized be the estimated pooled variance-covariance matrix, $\hat{\boldsymbol{\Sigma}}_{p l}$, which is defined as:

$$
\hat{\boldsymbol{\Sigma}}_{p l}=\frac{\left(n_{c}-1\right) \hat{\boldsymbol{\Sigma}}_{c}+\left(n_{h}-1\right) \hat{\boldsymbol{\Sigma}}_{h}}{n_{c}+n_{h}-2}
$$

where $\hat{\boldsymbol{\Sigma}}_{c}$ and $\hat{\boldsymbol{\Sigma}}_{h}$ are the unbiased estimators of the variance-covariance matrices in the current and healthy state. Unequal sample numbers for the estimation in the current and healthy state, $n_{c}$ and $n_{h}$, respectively, are also allowed. The $T^{2}(m)$ statistic follows Hotelling's distribution, $T_{m, n_{c}+n_{h}-2}^{2}$, with $m$ and $n_{c}+n_{h}-2$ degrees of freedom. This allows defining a hypothesis test of the $T^{2}(m)$ statistic by means of the $T_{m, n_{c}+n_{h}-2}^{2}$ cumulative distribution function, $F_{T_{m, n_{c}+n_{h}-2}^{2}}(1-\alpha)$, as:

$$
\begin{aligned}
& T^{2}(m) \leq F_{T_{m, n_{c}+n_{h}-2}^{2}}(1-\alpha) \quad \Rightarrow \quad H_{0} \text { is accepted } \\
& \text { Else } \quad \Rightarrow \quad H_{0} \text { is rejected }
\end{aligned}
$$

where $\alpha$ is the selected level of significance.

Under the assumption that the healthy state mean, $\boldsymbol{\mu}_{h}$, and variance-covariance matrix, $\boldsymbol{\Sigma}_{h}$, are known exactly or have been estimated very precisely and accurately, the availability of only a single sample of the DSF vector in the current state, $\hat{\mathbf{v}}_{c}$, allows calculating the better known squared Mahalanobis distance, $D^{2}(m)$, as a special case of the $T^{2}(m)$ statistic (Söderström and Stoica, 1989):

$$
D^{2}(m)=\left(\hat{\mathbf{v}}_{c}-\boldsymbol{\mu}_{h}\right)^{T} \boldsymbol{\Sigma}_{h}^{-1}\left(\hat{\mathbf{v}}_{c}-\boldsymbol{\mu}_{h}\right) \sim \mathcal{X}_{m}^{2}
$$

which follows a $\mathcal{X}_{m}^{2}$ distribution rather than general Hotelling's distribution, $T_{m, n_{c}+n_{h}-2}^{2}$. This statistic may be useful for rapid online SHM applications, when it is desired to obtain decisions about the current state as early as possible and abundant data exist for estimating healthy state statistical moments with high precision and accuracy.

A suitable objective function is required to identify and retain those features that improve damage detectability. Since we use a statistical hypothesis testing approach for making decisions about damage existence, a relative statistical distance is proposed for this task:

$$
T_{r e l}^{2}(m)=T^{2}(m) / F_{T_{m, n_{c}+n_{h}-2}^{2}}(1-\alpha)
$$


Division by the statistical threshold at a selected level of significance, $\alpha$, makes it possible to assess if the average contribution of the features to the statistical distance is sufficient to indicate damage, i.e. if $T_{r e l}^{2}(m)>1$. The optimal dimension of DSF vector, $m_{o p t}$, is found as the one that maximises the relative statistical distance, as it corresponds to the maximum separability between the healthy and damage case.

Even though, the contributions of individual DSFs to the statistical distance $T_{\text {rel }}^{2}(m)$ are not readily accessible because the cross-correlations entangle them with the remaining features, assessing their damage sensitivities separately can give an insight into potential optimal selections. The use of Fisher's criteria, $F C(i)$, as standardized distance between a univariate DSF, $v_{i}$, from the healthy and a selected reference damage state allows comparing damage sensitivities between different features. It can be calculated using the estimated means, $\hat{\mu}_{h, i}$ and $\hat{\mu}_{d, i}$, and pooled variances, $\hat{\sigma}_{p l, i}^{2}$, as

$$
F C(i)=\frac{\left(\hat{\mu}_{d, i}-\hat{\mu}_{h, i}\right)^{2}}{\hat{\sigma}_{p l, i}^{2}}
$$

Scaling with respect to a statistical threshold is not required because this is a constant as the degrees-of-freedom are the same.

\section{Principal component analysis}

DSF vectors, $\mathbf{v}_{i}$, of dimension $m$ and obtained from $n$ time series segments can be used to construct a DSF matrix, $\mathbf{V}$, of dimension $m \times n$ as:

$$
\mathbf{V}=\left[\begin{array}{llll}
\left(\mathbf{v}_{1}-\boldsymbol{\mu}\right) & \left(\mathbf{v}_{2}-\boldsymbol{\mu}\right) & \cdots & \left(\mathbf{v}_{n}-\boldsymbol{\mu}\right)
\end{array}\right]
$$

where zero mean column vectors of the DSF matrix, $\mathbf{V}$, are the result of removing the mean, $\mu$, common for all the DSF vector samples. PCA enables to transform the initial DSFs, $\mathbf{v}_{i}$, with the help of a linear transformation matrix, $\mathbf{T}$, into new linearly independent DSFs, $\mathbf{s}_{i}$, as:

$$
\mathbf{S}=\mathbf{T}^{T} \mathbf{V}
$$

where

$$
\mathbf{S}=\left[\begin{array}{llll}
\mathbf{s}_{1} & \mathbf{s}_{2} & \cdots & \mathbf{s}_{n}
\end{array}\right]
$$

$\mathbf{S}$ is the so-called score matrix of dimension $m \times n$. The $m \times m$ dimensional transformation matrix, $\mathbf{T}$, is obtained by performing singular value decomposition of the variancecovariance matrix, $\boldsymbol{\Sigma}$, of the initial DSFs, $\mathbf{v}_{i}$, as (Jolliffe, 2002):

$$
\boldsymbol{\Sigma}=\mathbf{T} \Lambda \mathbf{T}^{T}
$$

where $\boldsymbol{\Lambda}$ is a diagonal matrix containing the eigenvalues, $\lambda_{k}$, of $\boldsymbol{\Sigma}$ sorted in descending order. The order corresponds to the variances of the scores. The column vectors of the transformation matrix, $\mathbf{T}$, are the normalized eigenvectors called PCs and denoted as $\mathbf{t}_{k}$ :

$$
\mathbf{T}=\left[\begin{array}{llll}
\mathbf{t}_{1} & \mathbf{t}_{2} & \cdots & \mathbf{t}_{m}
\end{array}\right]
$$

The PCs, $\mathbf{t}_{k}$, describe an orthonormal projection leading to linearly independent variables, i.e. scores have a diagonal variance-covariance matrix.

\section{Algorithms for optimal feature selection}

FF selection is a method for identifying optimal selections of variables in statistical analysis, whose modest computational requirements, ease of implementation and short processing times are the main advantages. The identification is achieved by consecutively adding those DSFs that improve the objective function from all available DSFs. The DSFs associated with the maximum of the objective function are referred to the optimal selection. However, depending on the DSF vector dimensionality and the properties of the variance-covariance matrix of the DSFs in the healthy and reference damage state, the obtained result is not guaranteed to be the global optimum. Especially for high dimensional DSFs with strong correlations, there is risk of identifying only a local optimum by this procedure. However, the true optimum is guaranteed to be found independent of the feature dimension if the DSFs are uncorrelated. In case of weak correlations the FF procedure is also often unaffected. The latter case will apply when the PCA scores are used as DSFs. Here, we PC-transform both the healthy and damage state scores using the healthy state PCs, but the results suggest the diagonal structure of the variance-covariance matrix is not strongly altered in the damage state, making the FF method attractive.

A more general approach is the use of a global optimization algorithm for the selection of DSFs, which avoids the computationally heavy, or even prohibitive, assessing of all the possible DSF selections but enables to determine the true optimum with high confidence. GAs are derivative-free stochastic optimization techniques inspired by species evolutionary strategies at the gene level (Kramer et al., 2011). Different selections, or individuals, are represented by $m$-dimensional binary selection vectors, $\mathbf{b}_{i}$, where the $j$-th DSF is selected for $b_{i, j}=1$. In the first place, a parental population $\mathcal{P}$ of $\kappa$ individuals is randomly created. The actual optimization is then performed until a preselected number of generations is reached. (The traditional solution convergence criteria are not used because the binary GA operates in a discrete rather than a continuous solution space.) For new generations, dominant recombination of two randomly selected parents is employed to create $\gamma$ individuals of the offspring population $\mathcal{P}^{\prime}$. The mutation of each individual is done by a flip bit operation of $\beta$ randomly selected vector entries. The number of flipped entries describes the mutation rate. Then, the fitness function is evaluated for the union of both populations, $\mathcal{P} \cup \mathcal{P}^{\prime}$. It is defined as the product of the relative $T_{r e l}^{2}(m)$ statistic and a penalty term for similarity of solutions as

$$
f\left(\mathbf{b}_{i}\right)=T_{r e l}^{2}\left(m, \mathbf{b}_{i}\right) \times \min _{\mathbf{b}_{i} \in \mathcal{P}^{\prime \prime}}\left(1-S\left(\mathbf{b}_{i}, \mathbf{b}_{j}\right)\right)
$$

The penalty for similarity is calculated for the temporary set of already selected individuals, $\mathcal{P}^{\prime \prime}$. The similarity between the selection vectors is assessed with the help of the vector cosine distance, $1-S\left(\mathbf{b}_{i}, \mathbf{b}_{j}\right)$, (Singhal, 2001), where

$$
S\left(\mathbf{b}_{i}, \mathbf{b}_{j}\right)=\frac{\mathbf{b}_{i}^{T} \mathbf{b}_{j}}{\sqrt{\mathbf{b}_{i}^{T} \mathbf{b}_{i}} \sqrt{\mathbf{b}_{j}^{T} \mathbf{b}_{j}}}
$$

This is intended to prevent the selection of only similar individuals representing a local optimum. Finally, according 
to the Darwinian principle of survival of the fittest, $\kappa$ individuals with the highest fitness values are selected as parents for the next generation.

\section{Assessment of damage detection performance using receiver operating characteristic curves}

Although, the use of $T^{2}(m)$ statistics allows making decisions about the structural state with respect to a fixed statistical threshold, the performance of the presented damage detection method can also be investigated with the help of the so called receiver operating characteristics (ROCs) (Krzanowski and Hand, 2009). The concept is widely used in signal processing, medicine, biometrics and machine learning, and has also been adopted in damage detection studies (Mujica et al., 2014). It allows assessing the performance of a binary classifier independent of a selected fixed threshold value. Thus, a poorly or exceptionally well chosen threshold will not skew the assessment. ROC curves are obtained by plotting true positive rates against false positive rates for a wide range of classification thresholds. True positive rates are the relative ratios of the correctly classified true samples to the total number of positive samples in the set. On the other hand, dividing the number of samples incorrectly identified as positives in the set of all negative samples by the total number of true negatives gives the false positive rate. The range of thresholds to be investigated can be decided using all scores of the classifier to be evaluated, as suggested in Fawcett (2006).

Even though ROC curves enable to discuss the performance of different classifiers across a range of thresholds in detail, they may be impractical if many classifiers need to be assessed for different sensors or damage extents. A more concise performance measure will be useful. Therefore, the area under ROC curve (AUC) can be used for overall performance evaluation across the whole range of thresholds considered. The perfect classification performance has $\mathrm{AUC}=1.0$ and the random classification $\mathrm{AUC}=0.5$, respectively.

\section{Partial autocorrelation-based damage sensitive features}

The above discussions have used a generic DSF vector. However, to demonstrate practically the applicability of the proposed approach, PACCs of vibration response signals are selected for constructing the initial DSF vectors. A discrete univariate acceleration time series $x[t]$, where $t$ is a time instant, can be divided into $n$ segments, $x_{i}[t]$ ( $i=$ $1,2, \ldots, n)$, of common sample numbers. Each segment can be normalised by removing its estimated mean, $\hat{\mu}_{x_{i}}$, and dividing by its estimated standard deviation, $\hat{\sigma}_{x_{i}}$. The result are standard, zero-mean normally distributed time series segments, $z_{i}[t]$. For such a time series segment, the PACF, $\alpha_{i}[\tau]$, at lag $\tau$ can be defined as (Cryer and Chan, 2008):

$$
\alpha_{i}[\tau]=\operatorname{Corr}\left(z_{i}[t], z_{i}[t-\tau] \mid z_{i}[t-1], \ldots, z_{i}[t-\tau+1]\right)
$$

PACF at lag $\alpha_{i}[\tau]$ can be interpreted as the correlation between the shifted time series segments $z_{i}[t]$ and $z_{i}[t-$ $\tau]$ without effects of the intermediate variables $z_{i}[t-$ $1], \ldots, z_{i}[t-\tau+1]$, or conditional on those variables. The sample PACF, $\hat{\alpha}_{i}[\tau]$, can be efficiently computed by recursion as:

$$
\hat{\alpha}_{i}[\tau]=\frac{\hat{r}_{i}[\tau]-\sum_{k=1}^{\tau-1} \hat{a}_{i, k, \tau-1} \hat{r}_{i}[\tau-k]}{1-\sum_{k=1}^{\tau-1} \hat{a}_{i, k, \tau-1} \hat{r}_{i}[k]}
$$

where $\hat{r}_{i}[\tau]$ is the sample autocorrelation function of time series segment $z_{i}[t]$ at lag $\tau$, and $\hat{a}_{i, k, l}$ denotes the estimated $l$-th coefficient of a $k$-th order autoregressive model of the same segment. The above formula emphasises the close relationship between the PACF, autocorrelation function and autoregressive coefficients, which can be interchangeably estimated from one another (Kay, 1988). Another important common use of the PACF is as an indicator of the appropriate autoregressive model order (Box et al., 2008). Autoregressive processes are characterized by decay of the PACF, which is theoretically zero for lags $\tau$ higher than the required autoregressive model order, $p$. The PACF at lags higher than $p$ is approximately zero-mean Gaussian distributed with a variance (Quenouille, 1949)

$$
\sigma_{\hat{\alpha}_{i}[\tau]}^{2}=1 / n_{\text {samp }} \text { for } \tau \geqq p+1
$$

where $n_{\text {samp }}$ is the number of samples used for estimating the PACF. Knowing the approximate distribution allows selecting only significant PACCs to construct DSF vectors, $\mathbf{v}_{i}$, as

$$
\mathbf{v}_{i}=\left[\begin{array}{llll}
\hat{\alpha}_{i}[1] & \hat{\alpha}_{i}[2] & \cdots & \hat{\alpha}_{i}[m]
\end{array}\right]^{T}
$$

The above vectors are used in our research as full initial DSFs.

\section{Analytical problem illustration}

This section provides an example that illustrates the selection of DSFs for optimal damage detectability from amongst both initial and transformed DSF. A bivariate DSF vector is selected because it makes the example tractable manually and allows using two-dimensional visualizations as well as obtaining some exact formulas. However, the principles hold similarly for higher dimensional DSF vectors. The subscript $i$ referring to a specific time series segment is omitted for simplicity. It is assumed that the chosen DSF vector, $\mathbf{v}$, follows in the healthy state a bivariate Gaussian probability distribution, $\mathcal{N}\left(\boldsymbol{\mu}_{h}, \boldsymbol{\Sigma}_{h}\right)$, with a known true mean vector and variance-covariance matrix, $\boldsymbol{\mu}_{h}$ and $\boldsymbol{\Sigma}_{h}$, respectively. Knowledge of these characteristics enables to use a statistical test on the squared Mahalanobis distance (Equation (6)) to make a decision about the structural state using a single DSF vector of the current state, $\mathbf{v}_{c}$, as explained earlier. The $\mathcal{X}_{2}^{2}$ cumulative distribution function, $F_{\mathcal{X}_{2}^{2}}(1-\alpha)$, can be used to define a statistical threshold at a selected level of significance, $\alpha$.

For visualization of the corresponding space in two dimensions, the difference between the current state DSF vector using the initial features, $\mathbf{v}_{c}$, and the true mean in the healthy state, $\boldsymbol{\mu}_{h}$, is given as:

$$
\Delta \mathbf{v}=\left[\begin{array}{l}
v_{c, 1}-\mu_{h, 1} \\
v_{c, 2}-\mu_{h, 2}
\end{array}\right]=\left[\begin{array}{l}
\Delta v_{1} \\
\Delta v_{2}
\end{array}\right]
$$




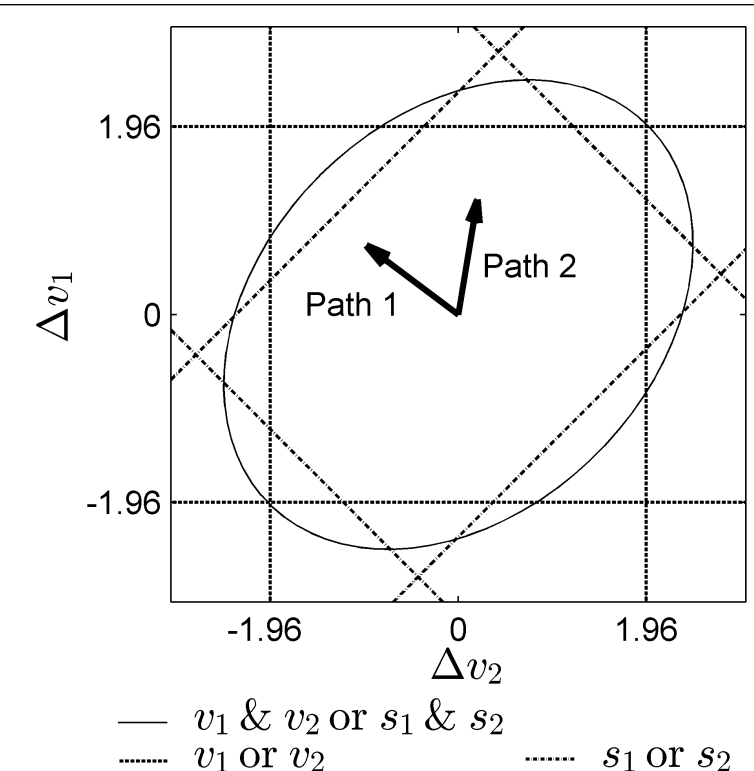

Figure 2. Regions of damage detectability for bivariate DSF with $\rho_{12}=0.3$.

and the variance-covariance matrix of the healthy DSF is

$$
\boldsymbol{\Sigma}_{h}=\left[\begin{array}{cc}
\sigma_{1}^{2} & \rho_{12} \sigma_{1} \sigma_{2} \\
\rho_{12} \sigma_{1} \sigma_{2} & \sigma_{2}^{2}
\end{array}\right]
$$

where the correlation between the two initial DSFs is quantified by the correlation coefficient, $\rho_{12}$. It can be further assumed for simplicity, but without loss of generality, that the standard deviations $\sigma_{1}$ and $\sigma_{2}$ are both equal to one. For a chosen statistical threshold, Equation (6) enables to define an elliptical region of acceptance of the null hypothesis in the initial DSF space as

$$
D^{2}(2)=\frac{\Delta v_{1}^{2}+\Delta v_{1}^{2}-2 \rho_{12} \Delta v_{1} \Delta v_{2}}{1-\rho_{12}^{2}} \leq F_{\mathcal{X}_{2}^{2}}(1-\alpha)
$$

This is shown in Figure 2 for a selected correlation coefficient $\left(\rho_{12}=0.3\right)$ and a level of significance of $5 \%$. It should be noted that the acceptance region becomes a circle for uncorrelated initial DSFs as a special case. Furthermore, the detectability regions when using either DSF $v_{1}$ or DSF $v_{2}$ only are also indicated. They appear as the horizontal and vertical band, respectively, in the initial feature space bounded by $\pm \sqrt{F_{\mathcal{X}_{1}^{2}}(1-\alpha)}$.

For the bivariate example, the PCs can be easily obtained as

$$
\mathbf{T}=\frac{\sqrt{2}}{2}\left[\begin{array}{cc}
1 & -1 \\
1 & 1
\end{array}\right]
$$

The transformation to scores using these PCs (Equation (10)) can be interpreted as observing the DSFs in a coordinate system rotated by $45^{\circ}$ clockwise. The null hypothesis acceptance region for using both PCA scores coincides with the elliptical acceptance region of both initial DSFs. However, the detection bands corresponding to the use of individual transformed DSFs appear as inclined, as shown in Figure 2. They are established with respect to the same statistical threshold of $\pm \sqrt{F_{\mathcal{X}_{1}^{2}}(1-\alpha)}$.
Two hypothetical damage scenarios are now considered, as indicated by Path 1 and 2 in Figure 2. These paths depict the loci of the DSFs as damage progresses. (Note that the paths do not have to be straight lines; for the purpose of this discussion their exact shape is of lesser importance than where they cross damage detection thresholds for different selections of DSFs.) Observing Path 1 leads to a conclusion that if both original DSF, $v_{1}$ and $v_{2}$, are used damage will be detected earlier than when using either $v_{1}$ or $v_{2}$ separately, because Path 1 crosses the ellipse earlier than any of the univariate damage detection boundaries. However, for Path 2 the situation is different: here using only $v_{1}$ detects damage earlier than using both $v_{1}$ and $v_{2}$. Furthermore, using only $v_{2}$ and assuming Path 2 continues as nearly vertical straight line as indicated in the figure one would detect this type of damage only very late (or never should Path 2 be exactly vertical). This comparison of Path 1 and 2 demonstrates that for some types of damages it is advantageous to use less than all the available DSFs - indeed using more DSFs only delays detection. However, these reduced DSF sets must be appropriately chosen and the selection depends on the type of damage, or its 'path'.

We now return to Path 1 and observe that if instead of using the original features one opts for the score corresponding to the second PC, damage will be detected earlier using this selection from the transformed features. This illustrates that beyond selecting optimal features from amongst the original ones, further improvements in early damage detection can be gained by transforming features using PCA. In fact, in Figure 2 one can see an octagon, laying fully inside the ellipse and defined by the damage detectability thresholds corresponding to using only one original or one PC-transformed feature. Thus, if one combines PCA and then selection of features, it is, in this case, always possible to improve damage detectability by using less than the full set of available original or transformed DSFs irrespective of the damage path.

The above discussion could have suggested that selecting a univariate DSF is always preferred to selecting a bivariate one. This, however, is not the case and in fact depends on the correlations between the original features, as shown in Figure 3 for $\rho_{12}=0.9$. Now the innermost area has no longer octagonal bounds because segments of the ellipse fall inside the octagon. Thus, for some types of damages, even if features are transformed using PCs, the optimal selection will include two features. It can actually be shown by using Equation (22) that this is going to be the case when the original feature correlation coefficient exceeds the critical value given as

$$
\rho_{12, \text { crit }}=1-8\left(\frac{\sqrt{F_{\mathcal{X}_{1}^{2}}(1-\alpha)}}{F_{\mathcal{X}_{2}^{2}}(1-\alpha)}-\frac{1}{2}\right)^{2}
$$

This critical value only depends on the ratio between the univariate and bivariate statistical thresholds at a chosen level of significance, $\alpha$. For $\alpha=5 \%$, used in our example, the critical correlation coefficient equals approximately 0.84 . 


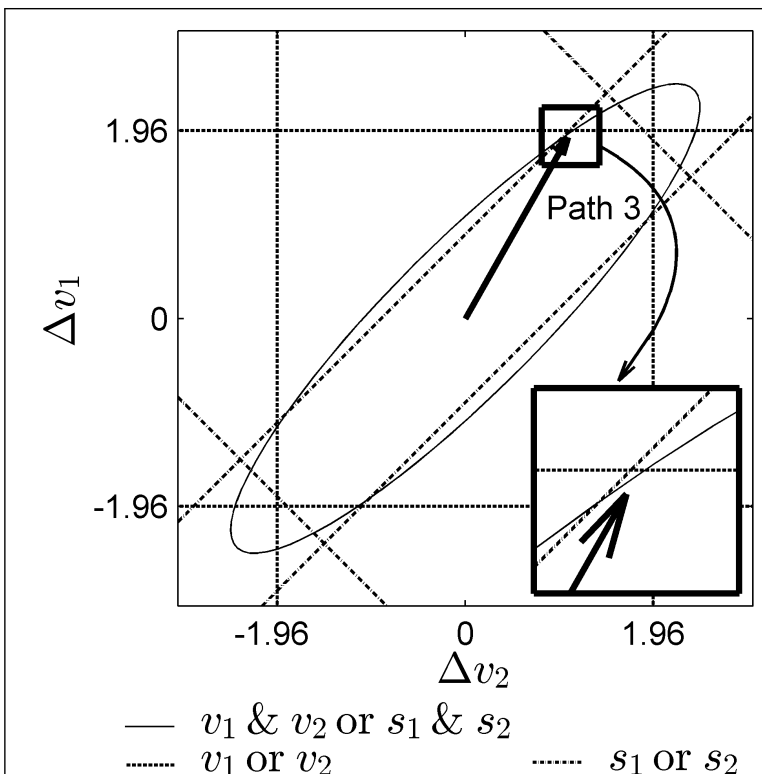

Figure 3. Regions of damage detectability for bivariate DSF with $\rho_{12}=0.9$.

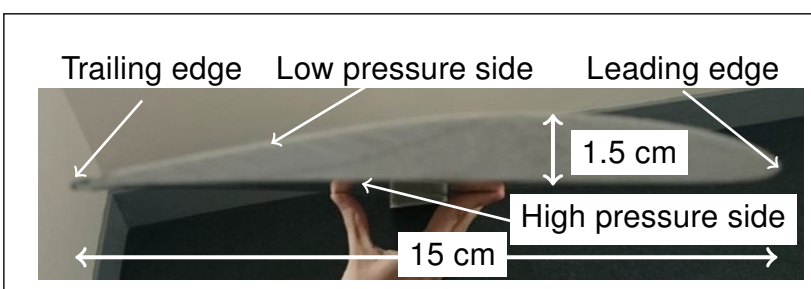

Figure 4. Experimental wind turbine blade cross-section.

\section{Experiments}

In order to demonstrate and verify the proposed concepts laboratory experiments were performed on a small WTB. This section describes first the configuration of the experimental set-up, then the dynamic modal characteristics of the WTB, and, finally, the determination of the PACF of dynamic responses as the preparation for the optimal DSF selection and actual SDD algorithm evaluation.

\section{Specimen and equipment}

The structure under study is the blade of a small wind turbine with $5 \mathrm{~kW}$ rated power output and rotor diameter of $5 \mathrm{~m}$. The WTB has a length of $2.36 \mathrm{~m}$. The cross-section is that of the aerofoil profile E387 with a constant width of $150 \mathrm{~mm}$, as shown in Figure 4. The profile has curved low-pressure side and flat high-pressure side. The solid WTB section is made by pultrusion of a glass-fibre reinforced epoxy composite. The total mass and mass density are estimated from measurements as $7,110 \mathrm{~g}$ and $2.30 \mathrm{~g} / \mathrm{cm}^{3}$, respectively.

For the experiments, a vertical configuration of the WTB is selected to save laboratory space. Cantilever-type boundary conditions, similar to those in real WTBs, are created by clamping the root to a massive steel base. This is shown in Figure 5. The base is sitting on a thick concrete floor to isolate it from uncontrolled ambient excitation sources.

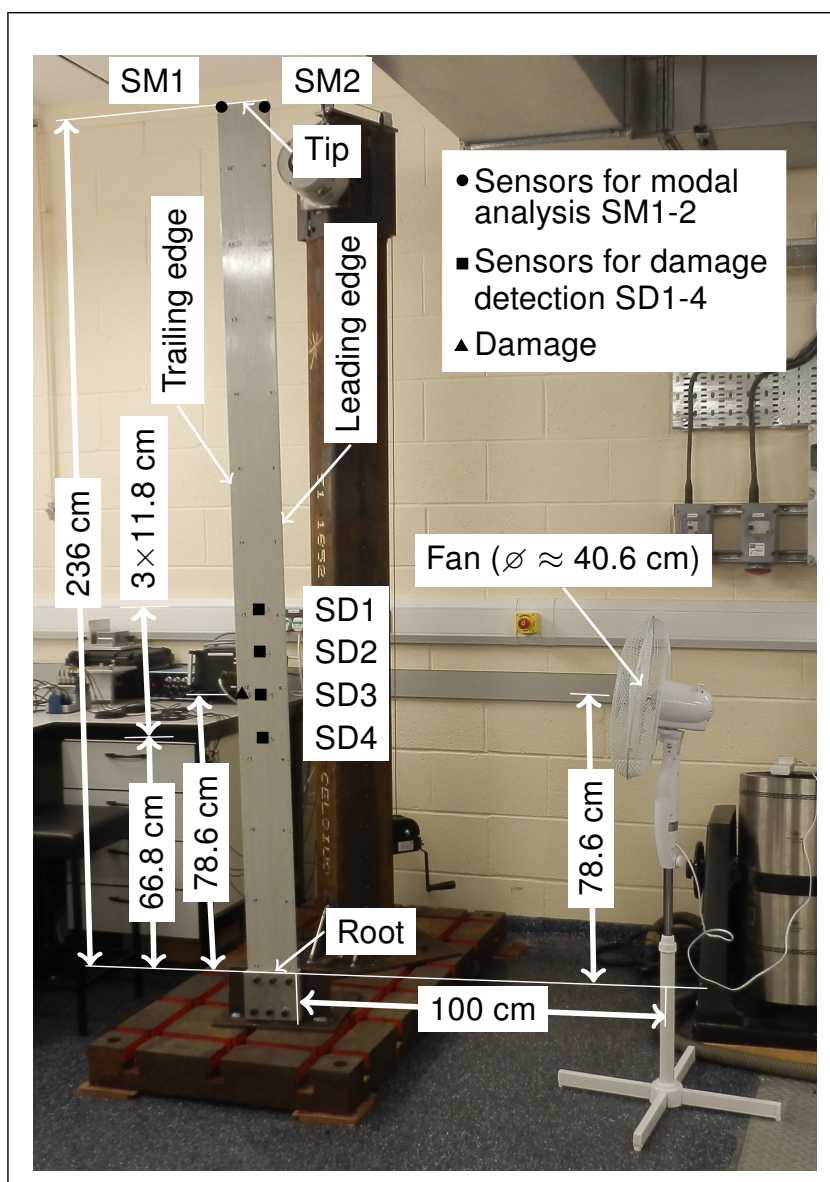

Figure 5. Experimental setup.

The WTB is excited in two ways. For experimental modal analysis, to determine the healthy WTB's modal dynamic characteristics an instrumented hammer model Brüel \& Kjær 8206 is used to apply impulse excitations at defined locations. The hammer has a sensitivity of $1.14 \mathrm{mV} / \mathrm{N}$, an effective mass of $100 \mathrm{~g}$, and can apply a maximum force of $4,448 \mathrm{~N}$. A soft polyurethane hammer tip is used, which enables to excite the WTB in a frequency range of up to $550 \mathrm{~Hz}$. The second method employed in the actual SDD study uses a household pedestal fan with a rotor diameter of $40.6 \mathrm{~cm}$ and height of $78.6 \mathrm{~cm}$ measured from the WTB root positioned at about $1 \mathrm{~m}$ from the WTB leading edge at an angle of attack of zero degrees (see Figure 5). In real life operations, this would correspond to feathered WTBs and stopped rotation typical for insitu inspections. The operating power can be selected in three levels with $40 \mathrm{~W}$ as the maximum. The use of a fan as non-contact excitation is advantageous because the structural system of the WTB is not affected, unlike when using a shaker connected with a stringer, which proved to modify markedly the boundary conditions. Furthermore, it allows the application of wind-like excitation mimicking that experienced in-situ.

Miniature piezoelectric accelerometers model Metra KS94B-100 with sensitivities of approximately $100 \mathrm{mV} / \mathrm{g}$ and frequency ranges of $0.5 \mathrm{~Hz}$ to $28 \mathrm{kHz}$ are used to measure the response accelerations. They are attached with adhesive wax to the WTB. Their locations are indicated in Figure 5 as SM1-2 for modal analysis and SD1-4 
Table 1. Identified modes of small scale WTB.

\begin{tabular}{ccc}
\hline Mode & Description & Frequency $[\mathrm{Hz}]$ \\
\hline 1 & $1^{\text {st }}$ flap-wise bending & 1.75 \\
2 & $2^{\text {nd }}$ flap-wise bending & 11.1 \\
3 & $3^{\text {rd }}$ flap-wise bending & 31.3 \\
4 & $1^{\text {st }}$ torsion & 38.8 \\
5 & $4^{\text {th }}$ flap-wise bending & 61.3 \\
\hline
\end{tabular}

for damage detection, respectively. Note that only sensors SM1-2 were attached during experimental modal analysis, and only sensors SD1-4 during SDD experiments. Force and acceleration signals are digitalized with two National Instruments NI-9234 data acquisition cards connected to a National Instruments cDAQ-9174 chassis and a laptop. Signal processing is performed with the National Instruments software LabView.

\section{Blade dynamic characteristics}

The dynamic characterization of the WTB is conducted by experimental modal analysis, where natural frequencies and mode shapes are estimated from accelerance frequency response functions (Ewins, 2000). A roving force and fixed response measurement configuration is selected. The measurement positions are shown in Figure 5. Two acceleration sensors, SM1 and SM2, are attached at the trailing and leading edge corners of the high-pressure side of the WTB tip for measuring flap-wise responses. This enables to capture all flap-wise and torsional modes in a given frequency range because these sensor locations do not correspond to nodes of cantilever beam modes. Impulse forces are applied at 7 equidistant locations along each WTB edge on the high-pressure side. Note that because impulse excitation was applied along both edges one sensor, either SM1 or SM2, would suffice to discern between bending and torsional modes, but two were used for cross checking the results. Readings of accelerations and forces are taken at a sampling rate of $2048 \mathrm{~Hz}$ for $30 \mathrm{~s}$ for each measurement. The identified modes are summarized in Table 1 . The low first natural frequency of $1.75 \mathrm{~Hz}$ demonstrates the high flexibility of the WTB.

\section{Experimental damage simulations}

Acceleration time series measurements for SDD are collected in the experimental configuration shown in Figure 5. The pedestal fan excites the structure continuously being set to the first power level. To avoid inflicting a permanent damage to the structure, change is introduced non-destructively by adding small masses at the trailing edge. For large real WTBs, the trailing-edge bondline is prone to damage. On the one hand, the production process, during which the upper and lower shells are bonded together, causes a reduction of structural resistance in that area. On the other hand, trailing-edge buckling adversely affects the structural performance of large WTBs (Jensen et al., 2011). Furthermore, inspections of 99 wind turbines with $100 \mathrm{~kW}$ and $300 \mathrm{~kW}$ rated power output revealed frequent failures at WTB trailing edges (Ataya and Ahmed, 2013). Motivated by the observation that the section with maximum chord was found to be exposed to that type of damage, the mass
Table 2. Non-destructive damage extent scenarios.

\begin{tabular}{ccc}
\hline Scenario & Absolute Mass [g] & Relative Mass [\%] \\
\hline 1 & 1 & 0.014 \\
2 & 2 & 0.028 \\
3 & 3 & 0.042 \\
4 & 4 & 0.056 \\
5 & 5 & 0.070 \\
6 & 6 & 0.084 \\
7 & 7 & 0.098 \\
8 & 8 & 0.113 \\
9 & 9 & 0.127 \\
10 & 10 & 0.141 \\
\hline
\end{tabular}

location for the experimental SDD tests is selected to be at approximately $33 \%$ of the WTB length from the root. This corresponds to the typical maximum chord location in large WTBs, where sharp geometrical changes from the circular root section to the aerofoil profile cause local stress concentrations. (However, it should be born in mind that our specimen is in fact a solid body with a prismatic crosssection.)

A steel washer of a mass of $5 \mathrm{~g}$ was glued onto the WTB at the selected damage location in order to be able to attach small magnets of known masses that simulate damage. The following healthy state measurements are taken with the washer attached. Then, up to 10 magnets, each of mass $1 \mathrm{~g}$, are added one by one to simulate different damage extents, as given in Table 2. Note that compared to the total mass of the WTB, even the largest added mass of $10 \mathrm{~g}$ is only $0.14 \%$ representing a small system modification.

The sensor layout is designed considering the chosen damage scenarios to place sensors close to the damage location, as shown in Figure 5. Four accelerometer sensors, denoted SD1-4, are placed on the centre line of the WTB's high-pressure side with different distances to the damage location. This allows obtaining insights into the interplay between detectable damage extents and sensor location and distances, which are significant characteristics for real life applications.

A typical response at sensor SD1 of the healthy WTB is shown in Figure 6 in both time and frequency domain. The time series shows significant fluctuations with amplitudes below $0.05 \mathrm{~g}$. Peaks at the identified natural frequencies in the power spectral density plot are also clearly visible, showing that excitation by the fan can generally provide good quality response signals.

\section{Partial correlation function identification and principal component analysis}

For the healthy state, the acquired acceleration response time series of $1,800 \mathrm{~s}$ is divided into 400 segments. Signals from the damage states, each of a $900 \mathrm{~s}$ duration, are split into 200 segments. In both cases, each segment is 5 s long with approximately $10 \%$ overlap. To account for possible excitation variations, each segment is normalized by removing its estimated mean and dividing by its estimated standard deviation. Then, each segment is filtered with a 


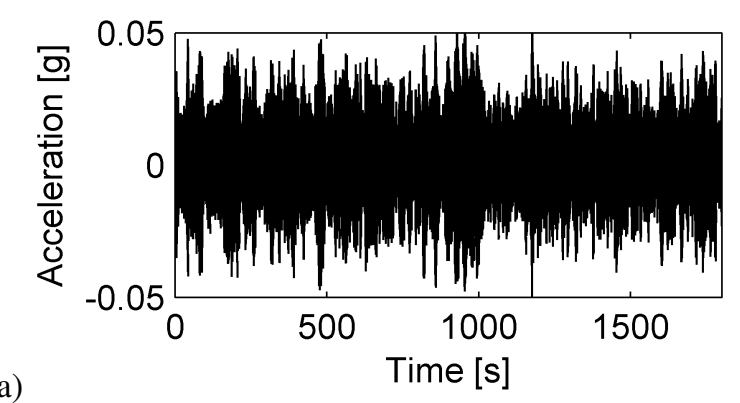

a)

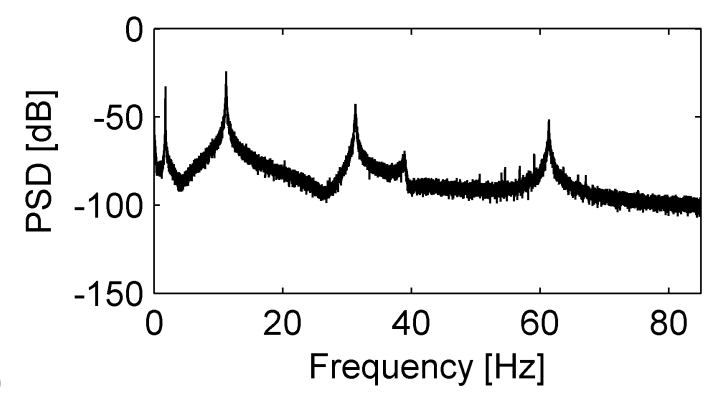

Figure 6. Response of the healthy WTB at sensor SD1: a) time series and b) power spectral densities.

Chebyshev Type I low-pass filter of order eight and a cutoff frequency of $204.8 \mathrm{~Hz}$ before being resampled at $256 \mathrm{~Hz}$, which corresponds to a decimation by a factor of 8 .

The healthy state measurements of the four sensors SD1-4 are used to identify an appropriate number of PACCs before they can be assumed to be zero. To do so, the PACFs of these signals are calculated for up to 100 lags. Mean values of each PACC are estimated from PACFs of the 400 segments. The results are shown in Figure 7 together with $95 \%$ confidence bounds for their values to be nil. The four PACFs have a common pattern with decreasing values for higher orders. Even though no clear cut-off is present in the PACFs, the amplitudes of PACCs for lags higher than 60 are noticeably smaller than the preceding ones. Furthermore, they generally fall within the $95 \%$ bounds for estimates from white noise process. Therefore, it is assumed that PACCs with lags from one to 60 are significant, and so they are selected for all sensor signals for the analyses that follow. These selections are used to construct the corresponding initial healthy state DSF matrices $\mathbf{V}_{h}$ (Equation (9)) for the four sensors.

The high initial DSF dimensionalities can adversely affect the damage detectability and increase computational burden. Therefore, PCA is used for transforming the correlated initial DSFs into uncorrelated scores. The explained variance of estimated PCs for sensor SD1 is shown in Figure 8 as an example. It can be seen that approximately the first 10 scores correspond to the highest variances, which are by two orders of magnitude larger than the smallest variances. The variance of scores larger than 10 shows only a small decay, while there is a noticeable drop for the last PC. The behaviour is almost identical for the remaining sensor results, thus their plots are omitted. However, this may not allow directly selecting the best PCs with respect to optimal damage detectability.

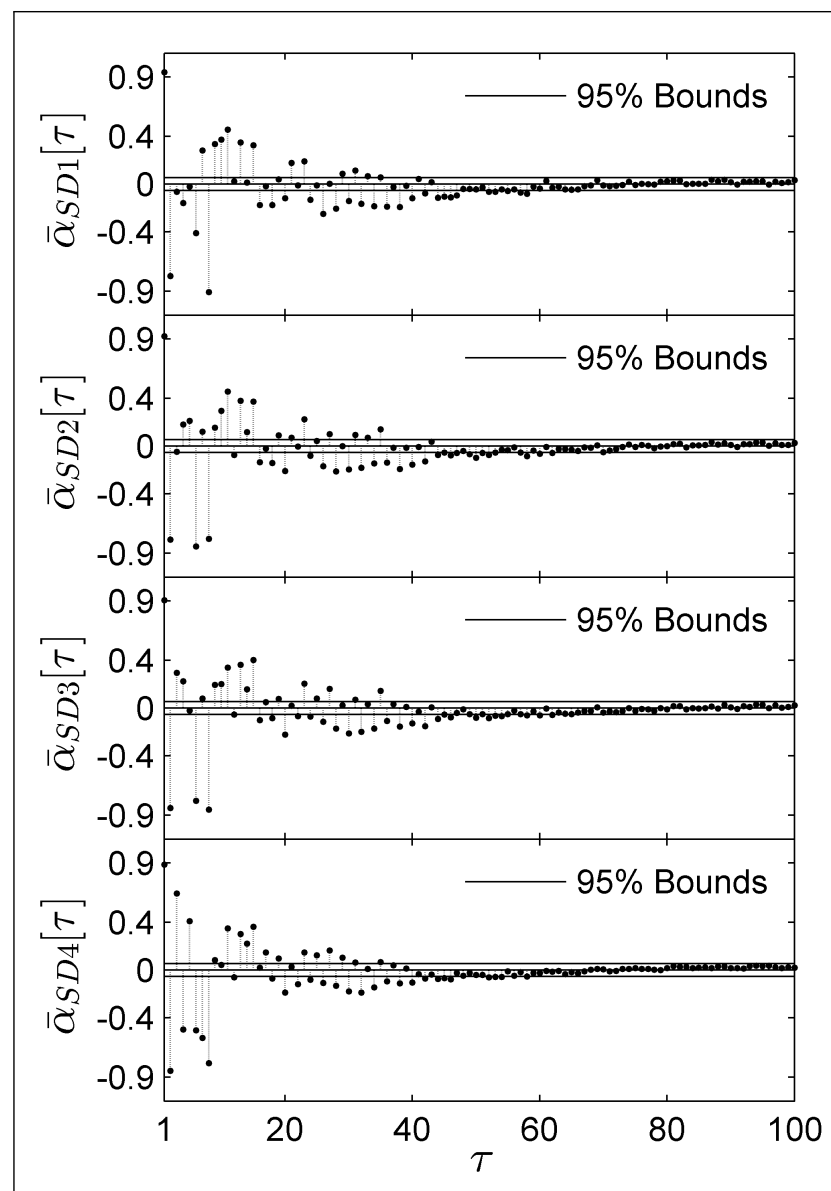

Figure 7. Estimated mean of partial autocorrelation functions (PACFs) in healthy state for sensors SD1-4.

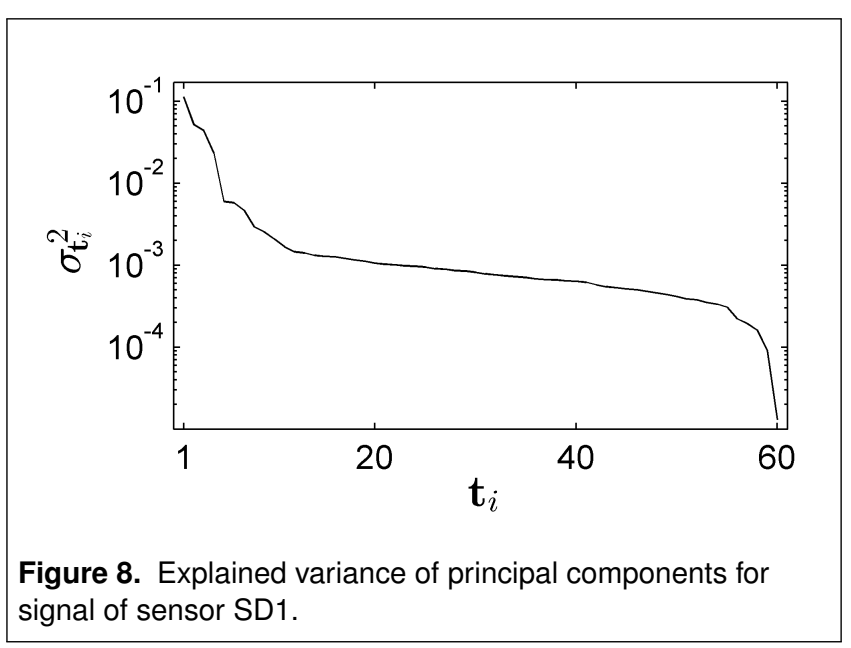

\section{Structural damage detection}

This section shows first the analyses and process leading to the identification of optimal selections from the initial and transformed DSFs that enhance damage detectability. This is done for a selected damage extent case, referred to as the reference damage case. The efficiency of the FF and GA algorithms is investigated and compared. Then, SDD results using the optimal DSF selections, and also the full set of DSFs for comparison, are presented and discussed for varying damage extents using the remaining 9 simulated damage cases, and previously unseen data from the reference damage and healthy cases. Additionally, the effect of DSF 


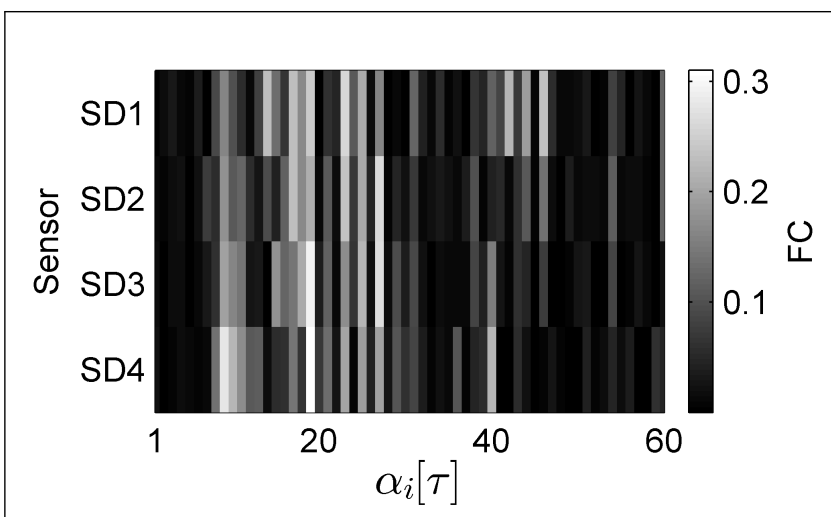

Figure 9. Damage sensitivity of initial features (PACCs) by Fisher's criterion.

small sample size is considered and the influence of varying damage detection thresholds is investigated. Results across all fours sensors are compared.

\section{Identification of optimal damage sensitive features}

Assessing univariate DSFs separately can give a first insight into their damage sensitivities. Therefore, FCs for PACC estimates of the four different sensor signals are shown in Figure 9. They are calculated between the healthy state and a reference damage state with $5 \mathrm{~g}$ attached magnet mass for lags from one to 60 . There is a common pattern identifiable for all four sensors with high sensitivities for PACCs seven to 31 and 36 to 46 , although their appearance is relatively unstructured in these ranges. However, results between sensors are comparable. Figure 10 presents sensitivities for the same reference damage state as Fisher's criteria calculated for scores obtained by transforming the initial features by the healthy state PCA transformation matrix. The obtained sensitivity patterns are markedly different compared to those of the initial DSFs. The highest sensitivities are mainly concentrated in two scores for each sensor. For all four sensors, score 59 is strongly affected by the introduced damage (for sensors SD2-4 the strongest). The other strongly affected score is 58 for sensors SD1 and SD4, in contrast to score 57 for sensors SD2 and SD3. The fact that the most damage affected scores are these with the highest numbers can be explained by the fact that they have small variances in the healthy state and thus even small damage related shifts in DSFs easily make them stand out. Comparing the results for the two different DSF types, the initial and the transformed, illustrates the advantages of using PCA scores. Here, a smaller number of the transformed DSFs is affected by damage but their sensitivities to damage are higher than for the initial PACCs. This indicates that linear combinations of the initial univariate DSFs defined by PCs may yield higher damage sensitivities than using selected univariate DSFs. Nonetheless, investigating the interplay between statistical thresholds and the actual multivariate selections, captured by the relative distance $T_{r e l}^{2}(m)$ (Equation (7)), is required for making decisions about the optimal DSF selection.

The FF and GA-based feature selection procedures are applied to both DSF types estimated from the healthy state

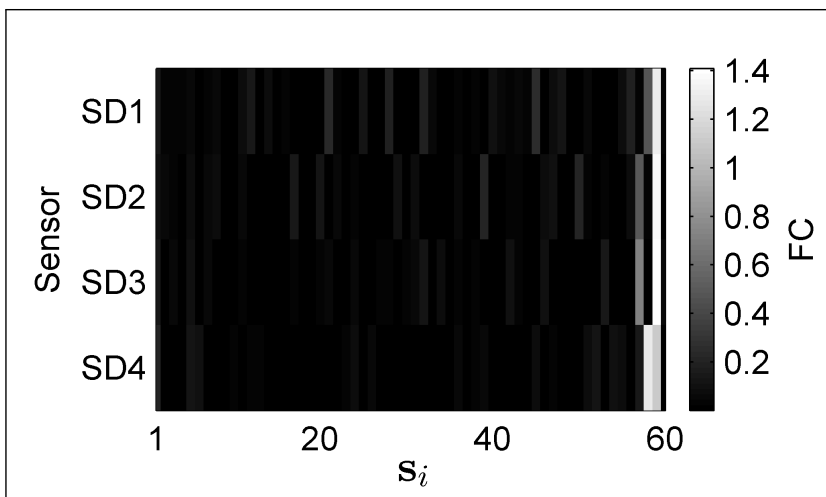

Figure 10. Damage sensitivity of transformed features (PCA scores) by Fisher's criterion.

and the reference damage state with a $5 \mathrm{~g}$ mass. In the case of initial DSFs, sample means and variance-covariance matrices are calculated for PACCs from lag one to 60 estimated from the 400 pre-processed time series segments in the healthy state and 200 segments in the reference damage state. In FF, DSFs are ranked by incrementally adding one by one those DSFs that increase the corresponding $T_{r e l}^{2}(m)$ statistic the most. Then, the optimal selection is identified as that where $T_{\text {rel }}^{2}(m)$ peaks. Table 3 shows the selection results for all four sensors with the corresponding maxima of the relative statistic. Additionally, the $T_{r e l}^{2}(m)$ statistic for the full set of 60 PACCs (or all 60 PC scores) is given as benchmark. It can be seen that the obtained PACC selections for the four sensors differ significantly in the number of the selected DSFs from 28 PACCs down to only one PACC for sensors SD1 and SD4, respectively. Nevertheless, the $T_{r e l}^{2}(m)$ statistics, as a measure of damage detectability, match closely for all sensors with a range between 12.40 and 14.89. The FF selection achieves the highest detectability for sensor SD4 and the lowest for sensor SD1. Comparing these results to the performance of the full feature set reveals improvements by a factor of two. Contrary to the FF selections, the highest detectability for the full set of PACFs is given by sensor SD1, and results of sensor SD3 correspond to the lowest.

The next selection method for initial DSFs defined by PACFs uses a GA for identifying optimal selections from different sensor signals. For this process, 8 parental and 20 binary offspring selection vectors were used. The mutation rate was defined by flipping only one entry for each offspring individual. This was kept constant for the entire search process. The algorithm was run 100 times with different random initial parental populations. The best DSF selections from the 100 runs are also given in Table 3. The selections obtained by FF and GA are equal for sensors SD3 and SD4, while there are differences for the remaining sensors. The selections estimated for sensor SD2 differ in two PACCs, where using the GA leads to a slightly higher $T_{r e l}^{2}(m)$ statistic of 13.59 compared to 13.50 for the FF selection. For sensor SD1, the GA selection represents a subset of 7 PACCs from the previous 28 strong FF selection. Nevertheless, the $T_{\text {ref }}^{2}(m)$ statistic is $6 \%$ higher for this subset.

Scores are calculated with the help of transformation matrices defined by PCA of the variance-covariance matrix 
Table 3. Optimal feature selections using reference damage extent of $5 \mathrm{~g}$ added mass.

\begin{tabular}{cccccc}
\hline & & & & \multicolumn{2}{c}{$T_{r e l}^{2}(m)$} \\
Sensor & Feature Type & Selection Method & Selected Feature(s) & Selection & Full Set \\
\hline SD1 & PACF & FF & $1,9,14-32,40-44,46,60$ & 12.40 & 7.59 \\
SD1 & PACF & GA & $14,19,22,42-44,46$ & 13.14 & 7.59 \\
SD1 & Scores & FF/GA & 59 & 45.29 & 7.59 \\
SD2 & PACF & FF & $23,27,28$ & 13.50 & 6.69 \\
SD2 & PACF & GA & $19,27,28,43$ & 13.59 & 6.69 \\
SD2 & Scores & FF/GA & 59 & 47.24 & 6.69 \\
SD3 & PACF & FF & $15,19-28,40,43$ & 12.44 & 5.96 \\
SD3 & PACF & GA & $15,19-28,40,43$ & 12.44 & 5.96 \\
SD3 & Scores & FF/GA & 59 & 48.74 & 5.96 \\
SD4 & PACF & FF & 19 & 14.89 & 6.59 \\
SD4 & PACF & GA & 19 & 14.89 & 6.59 \\
SD4 & Scores & FF/GA & 58,59 & 55.23 & 6.59 \\
\hline
\end{tabular}

of the healthy state PACF estimates with lags one to 60 . The same transformation matrix is used for the healthy and reference damage state, where in both cases the mean DSF vector estimates of the healthy state are subtracted from the DSF vectors. Then, score vector entries were selected by the FF and GA algorithm. Due to only small differences between the variance-covariance matrix structures of DSFs in both states, the scores are only weakly correlated for the reference damage state. Therefore, the FF and GA lead to identical selections for all the sensors. Comparing the numbers of selected initial DSFs above to the numbers of transformed DSFs, with only one or two selected PC scores illustrates how damage sensitivity is condensed in an extremely limited number of transformed features. Furthermore, the corresponding $T_{\text {rel }}^{2}(m)$ statistics are substantially higher for all sensors using the selected PC scores. The highest $T_{r e l}^{2}(m)$ statistic, 55.23 is obtained for sensor SD4. Sensor SD2 gave the poorest performance, but $T_{r e l}^{2}(m)$ is still equal to 45.29. These damage detectability indices represent a dramatic improvement of a factor of approximately three to four compared to the initial DSF optimal sets, and of a factor of approximately six to eight compared to when all initial DSFs are employed.

The optimal DSF selection results can be summarized as follows. The full set of DSFs leads in all cases to the lowest $T_{r e l}^{2}(m)$ statistics. However, all of them are higher than one, thus the statistical detection threshold is exceeded using statistical estimates obtained from 200 current state DSF vectors. The results obtained for FF and GA selections have comparable $T_{\text {rel }}^{2}(\mathrm{~m})$ statistics for the initial DSFs and are identical for the PCA scores. This indicates that in the present problem the simple FF algorithm allows identifying efficient DSF selections, which avoids the substantially higher computational effort of GA-based selections. Furthermore, the use of PCA scores enables to improve the damage detectability significantly with respect to the initial DSF selections and the full set of PACCs. Thus, using PC scores selected by FF is a promising approach for improving the detectability of early damages with only limited additional computational effort.

\section{Structural damage detection performance}

The performance of the proposed damage detection algorithm using the optimal DSF selections established earlier will now be studied. The performance tests are intended to be challenging by considering previously unseen healthy datasets and damage cases, where only extremely limited numbers of samples are used for determining the actual structural state. Additionally, the performance is evaluated over a range of damage detection thresholds, unlike in the training stage when a single threshold was used.

The $T^{2}(m)$ statistics allow accounting for estimates of the mean and variance-covariance matrices obtained from different numbers of samples in the healthy and current state. In practical applications, the number of samples to be used will depend on their availability and on how quickly one wants to detect an altered structural state. For example, if early decision making is desired then the use of a small sample size in the current state may be necessary. However, there is a trade-off between the damage detectability and the sample numbers and smaller samples, with the associated heightened uncertainties, can impact adversely damage detectability. For the present study, it is assumed that detailed information is available in the healthy state by using all the available 400 samples. This is a realistic assumption as a wind turbine will normally be in operation in its healthy state for an extended period of time permitting adequate data collection. Then, for making decisions about the current structural state, only single DSF vectors are drawn from all available samples in the current state, i.e. 400 or 200 for the unseen healthy or each damage test datasets, respectively. The variance-covariance matrix of this state is then not required for calculating the $T_{r e l}^{2}(m)$ statistics. This is applied to all sensor signals of an unseen healthy measurement and all the damage extent scenarios.

In the present case, classification performance is assessed using ROCs with classification thresholds chosen as the values of the $T_{r e l}^{2}(m)$ statistic. The classifiers use the different optimal DSF selections indicated by the FF method and GA from amongst the original and transformed DSFs, and the full set of features is also included. Binary classification is attempted, where one class is the healthy state and the other class corresponds to a selected damage state. The ranges of threshold values in our case were obtained as ordered $T_{r e l}^{2}(m)$ statistics of actual features corresponding to the two classes.

Figure 11 shows the results for the three selected DSF subsets and the full set of PACCs of sensor SD1 for 


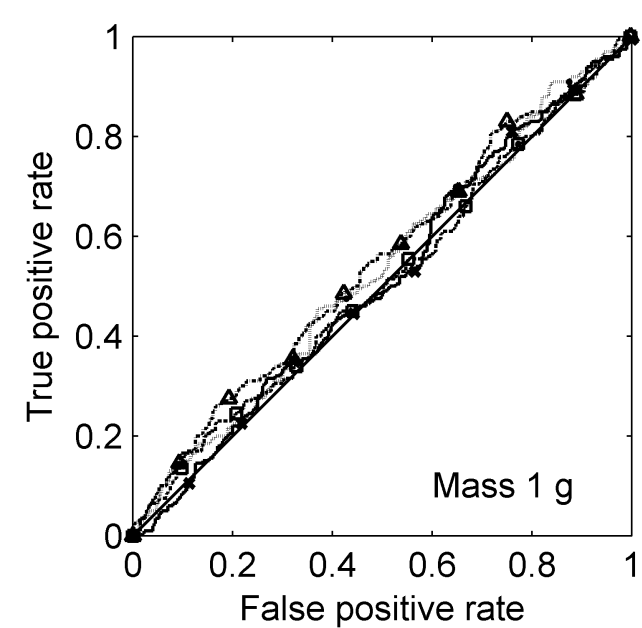

a)

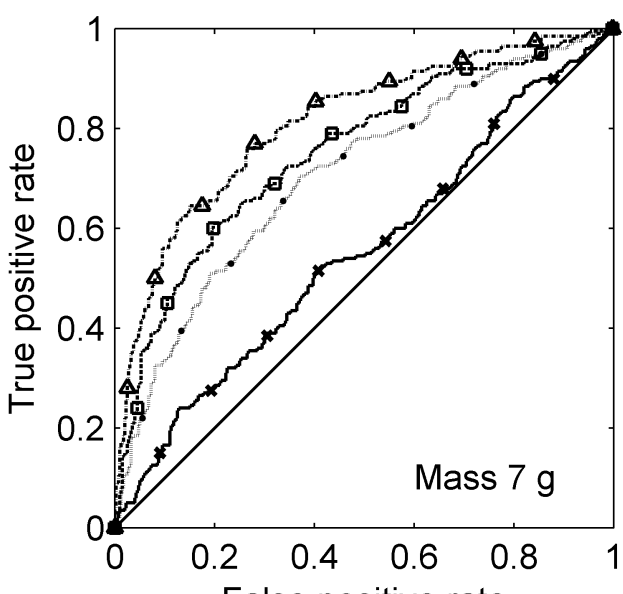

c) False positive rate

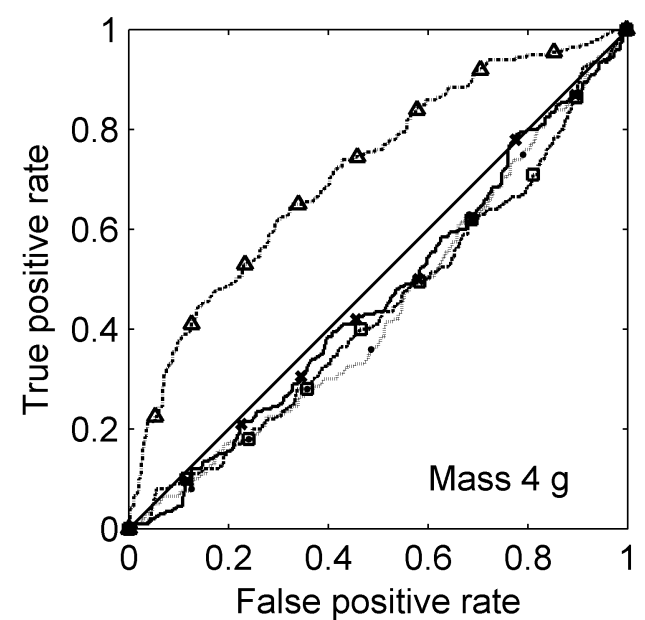

b)

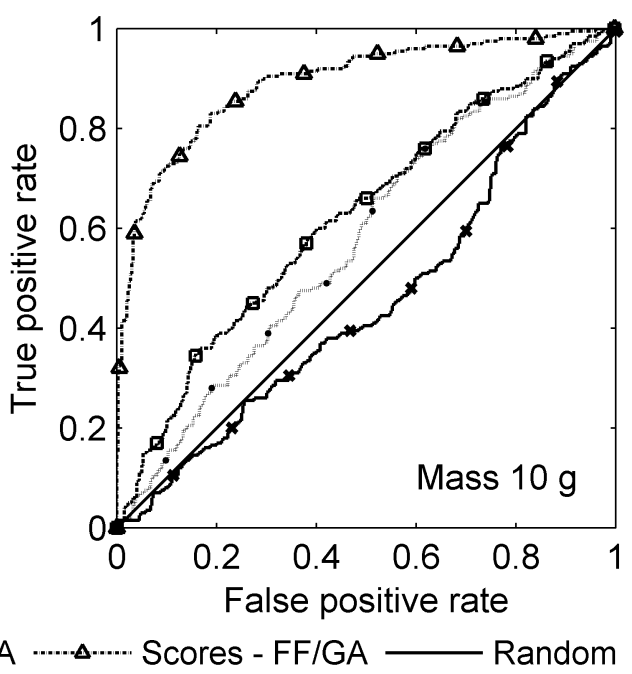

Figure 11. Receiver operating characteristic curves of optimal DSF selections for selected damage extents: a) $1 \mathrm{~g}$, b) $4 \mathrm{~g}$, c) $7 \mathrm{~g}$ and d) $10 \mathrm{~g}$.

damage cases of $1 \mathrm{~g}, 4 \mathrm{~g}, 7 \mathrm{~g}$ and $10 \mathrm{~g}$. A straight diagonal line is also drawn which is the ROC of a purely random classifier with $50 \%$ chance of obtaining a correct result. A perfect classifier, on the other hand, would have a bilinear ROC starting at true positive rate $=1$ and false positive rate $=1$ for very small detection thresholds, going through true positive rate $=1$ and false positive rate $=0$ and terminating at true positive rate $=0$ and false positive rate $=0$ for large detection thresholds. This ideal performance can be visualized by considering classifications obtained with detection thresholds sweeping between zero and a large value for two well-separated clusters with very small intercluster spreads and corresponding to two different states. It can be seen in Figure 11 that for the damage simulated by attaching $1 \mathrm{~g}$ mass all the DSF selections lead to similar results close to those of a random classification. This can be explained by the fact that adding $1 \mathrm{~g}$ does not change the structural system enough to overcome noise in the data and detect damage. However, for the next damage case of $4 \mathrm{~g}$, the ROC curve of the optimally selected PCA scores provides significantly better performance compared to the random classifications, while the initial full DSF set and the selections from the full set do not show improved performance. In fact, their performance is even slightly less than random, indicating confused classifiers. Similarly, the selected transformed DSFs outperform the other DSFs for $7 \mathrm{~g}$ of attached mass, although in this case some of them (the FF selection and the full set of PACCs) come close. The GAbased selection does not enable to distinguish between the two structural states. For $10 \mathrm{~g}$ of attached mass, the selected scores are superior with the corresponding ROC curve even closer to the ideal classifier then in the previous cases. The remaining feature sets are here close to the random performance.

Additionally to ROCs, AUCs allow for a more concise representation of the performance of different classifiers applied to different datasets. Figure 12 shows the AUCs for all combinations of DSF selections, sensors and damage extents. The above discussion of ROC curves can be easily verified. However, knowledge of the AUCs for all the damage extents enables to assess another important property of classifiers, i.e. how their damage detectability performance changes with increasing damage extent or severity. Ideally, there should be a monotonic improvement. Figure 12 reveals that damage detection performance of the classifiers based on PACFs selections and the full set of 


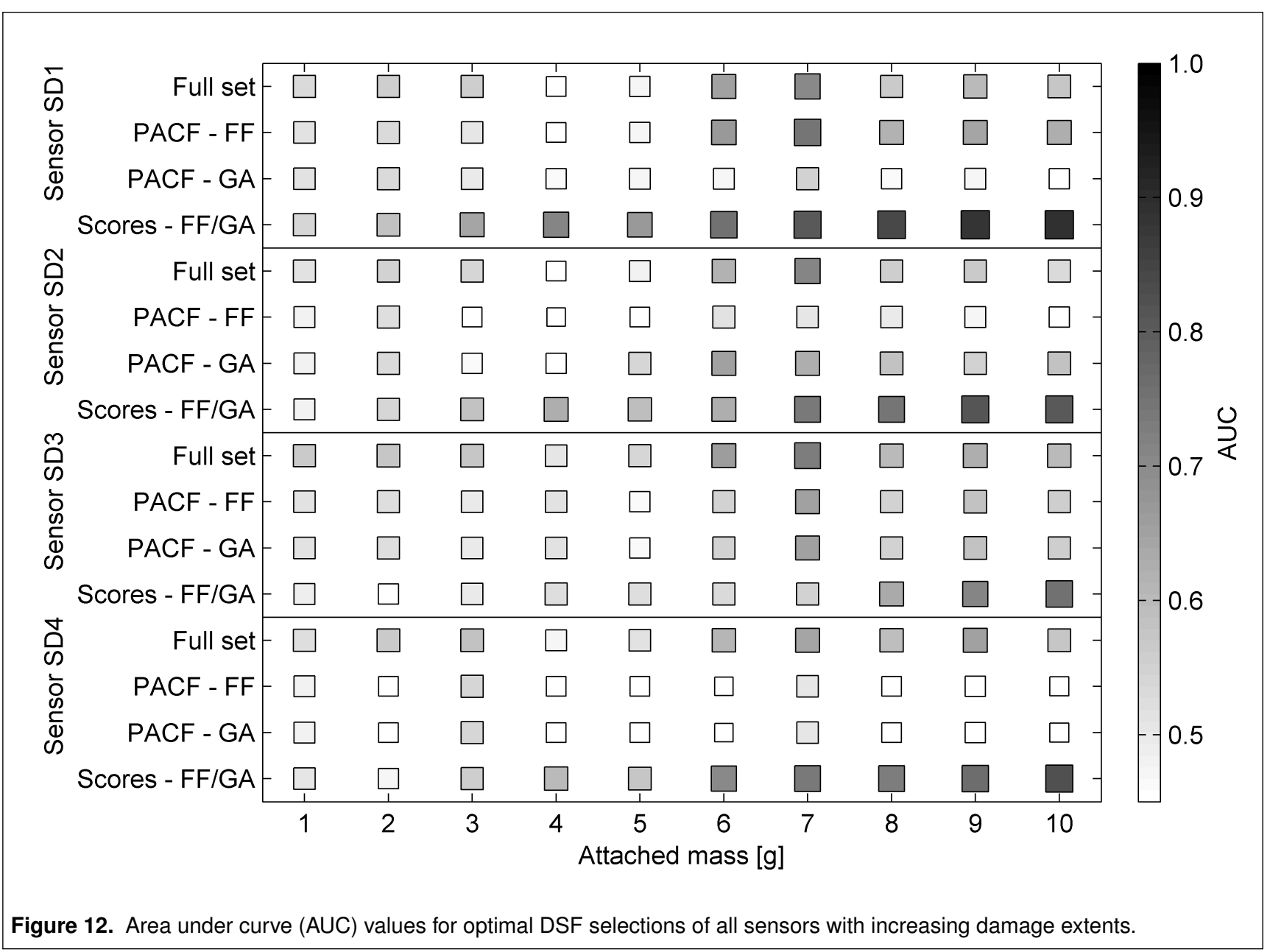

coefficients does not show the desired behaviour. The AUCs of full PACC sets first drop between the $3 \mathrm{~g}$ and $4 \mathrm{~g}$ mass cases, then they increase to the maxima for $7 \mathrm{~g}$, and then drop again. This behaviour can be observed for all sensors. Furthermore, the PACC subsets obtained using the FF or GA selections behave comparably, although AUCs have lower values. For sensor SD1, GA-based selection leads, on average, to lower AUC values for damage extents above 5 g compared to the FF subset. Signals from sensor SD2 result in the opposite behaviour. However, scores from the selected PCs show a similar desirable monotonic pattern for all four sensors with AUCs increasing with the attached mass (with a small exception of sensors SD3 and SD4 for $1 \mathrm{~g}$ and $2 \mathrm{~g}$ ), where the overall best detection performance is achieved for sensor SD1 and $10 \mathrm{~g}$ mass. Over the full range of damage extents, the selected PC scores of sensor SD1 show the best performance compared to other sensors. The poorest performance for the selected PCA scores is for sensor SD3. This additional evaluation conforms that the optimal selection from PC transformed initial DSFs outperforms all the other alternatives considered.

\section{Conclusions}

This paper has discussed the problem of optimal selection of DSFs for their application to SDD. Initial DSFs were defined as PACCs estimated from vibration responses. Scores obtained by PCA of these initial DSFs were proposed as transformed DSFs. The optimality problem was formulated as the selection of a subset of either initial or transformed DSFs to maximize a statistical distance between DSFs of the healthy and a reference damage state compared to a statistical threshold. The FF and GA selection procedures were proposed for identifying the optimal selections of DSFs. Experiments were performed on a small WTB made of a composite material, whose modal characteristics were identified by experimental modal analysis using an instrumented hammer. Then, a domestic fan was used to excite the WTB in a non-contact way resembling actual operating conditions. Non-destructive damage scenarios were simulated by attaching small masses at the WTB's trailing-edge. The measured acceleration signals were used to estimate PACFs at several sensor locations. For both DSF types, FF selection enabled to identify DSF subsets comparable or identical to the GA-based selections while being computationally more efficient. These attempts to identify optimal DSF subsets from the single reference damage case led to a conclusion that the use of PCA scores can dramatically reduce the DSF dimensionality, while simultaneously increasing the separation between the features from the healthy and damage case. While the use of the optimal selections from amongst the initial features also showed visible benefits, those were markedly less compared to the PCA scores. Damage classifiers using the optimal DSFs selected based on the single reference damage state were then subjected to challenging evaluation tests using previously unseen data from the healthy state and a range of damage extents. Their performance was also checked 
across a wide range of damage detection thresholds using ROC curves and effects of using a limited sample size were additionally considered. These tests showed that only the PCA score selections stood their ground quite well, while the remaining ones often performed similarly to a random classifier or even worse. Furthermore, the use of the optimal set of the PCA scores led to the desirable robust monotonic increase of damage detectability with its extent. Thus, the approach comprising PACF time series modelling with statistical hypothesis testing of PCA scores obtained from the FF selection procedure is promising for future applications in vibration-based SDD. Future research will explore the use of other linear and non-linear transformations applied to DSF which may further enhance damage detectability and ensure features change monotonically with increasing damage extent. An attempt to explore optimisation of DSFs for detection of wider ranges of damage extent scenarios should also be undertaken as the current research indicates the classifier performance may suffer when exposed to new cases. The influence of environmental and operational factors on the optimality should also be investigated and methods to deal with those developed if required. Finally, it will be worthwhile to extent the methodology to address the task of damage localization.

\section{Acknowledgements}

Piotr Omenzetter and Simon Hoell's work within the Lloyd's Register Foundation Centre for Safety and Reliability Engineering at the University of Aberdeen is supported by Lloyd's Register Foundation. The Foundation helps to protect life and property by supporting engineering-related education, public engagement and the application of research.

\section{References}

Ataya S and Ahmed MMZ (2013) Damages of wind turbine blade trailing edge: Forms, location, and root causes. Engineering Failure Analysis 35: 480-488.

Bandara RP, Chan THT and Thambiratnam DP (2014a) Frequency response function based damage identification using principal component analysis and pattern recognition technique. Engineering Structures 66: 116-128.

Bandara RP, Chan THT and Thambiratnam DP (2014b) Structural damage detection method using frequency response functions. Structural Health Monitoring 13(4): 418-429.

Benedetti M, Fontanari V and Zonta D (2011) Structural health monitoring of wind towers: Remote damage detection using strain sensors. Smart Materials and Structures 20(5): 1-13.

Beyer HG and Schwefel HP (2002) Evolution strategies - A comprehensive introduction. Natural Computing 1(1): 3-52.

Blanco MI (2009) The economics of wind energy. Renewable and Sustainable Energy Reviews 13(6-7): 1372-1382.

Box GEP, Jenkins GM and Reinsel GC (2008) Time Series Analysis: Forecasting and Control. 4th edition. Hoboken, USA: Wiley.

Brownjohn JMW, de Stefano A, Xu YL, Wenzel H and Aktan AE (2011) Vibration-based monitoring of civil infrastructure: Challenges and successes. Journal of Civil Structural Health Monitoring 1(3-4): 79-95.

Carden EP and Brownjohn JMW (2008) Fuzzy clustering of stability diagrams for vibration-based structural health monitoring. Computer-Aided Civil and Infrastructure Engineering 23(5): 360-372.

Carden EP and Fanning P (2004) Vibration based condition monitoring: A review. Structural Health Monitoring 3(4): 355377.

Chakraborty D, Kovvali N, Papandreou-Suppappola A and Chattopadhyay A (2014) An adaptive learning damage estimation method for structural health monitoring. Journal of Intelligent Material Systems and Structures 26(2): 125-143.

Chen B and Zang C (2011) A hybrid immune model for unsupervised structural damage pattern recognition. Expert Systems with Applications 38(3): 1650-1658.

Chen H, Kurt M, Lee YS, McFarland DM, Bergman LA and Vakakis AF (2014) Experimental system identification of the dynamics of a vibro-impact beam with a view towards structural health monitoring and damage detection. Mechanical Systems and Signal Processing 46(1): 91-113.

Ciang CC, Lee JR and Bang HJ (2008) Structural health monitoring for a wind turbine system: A review of damage detection methods. Measurement Science and Technology 19(12): 1-20.

Cross EJ, Manson G, Worden K and Pierce SG (2012) Features for damage detection with insensitivity to environmental and operational variations. Proceedings of the Royal Society A: Mathematical, Physical and Engineering Sciences 468(2148): 4098-4122.

Cryer JD and Chan KS (2008) Time Series Analysis with Applications in R. New York, USA: Springer.

da Silva S, Dias Junior M, Junior VL and Brennan MJ (2008) Structural damage detection by fuzzy clustering. Mechanical Systems and Signal Processing 22(7): 1636-1649.

de Lautour OR and Omenzetter P (2010a) Damage classification and estimation in experimental structures using time series analysis and pattern recognition. Mechanical Systems and Signal Processing 24(5): 1556-1569.

de Lautour OR and Omenzetter P (2010b) Nearest neighbor and learning vector quantization classification for damage detection using time series analysis. Structural Control and Health Monitoring 17(6): 614-631.

Doebling SW, Farrar CR and Prime MB (1998) A summary review of vibration-based damage identification methods. Shock and Vibration Digest 30(2): 91-105.

Doebling SW, Farrar CR, Prime MB and Shevitz D (1996) Damage identification and health monitoring of structural and mechanical systems from changes in their vibration characteristics: A literature review. Technical Report LA13070-MS, Los Alamos National Laboratory, Los Alamos, USA.

Döhler M, Marin L, Bernal D and Mevel L (2013) Statistical decision making for damage localization with stochastic load vectors. Mechanical Systems and Signal Processing 39(1-2): 426-440.

Ewins DJ (2000) Modal testing: Theory, practice and application. Hoboken, USA: Wiley-Blackwell.

Fan W and Qiao P (2011) Vibration-based damage identification methods: A review and comparative study. Structural Health Monitoring 10(1): 83-111.

Farhidzadeh A, Salamone S and Singla P (2013) A probabilistic approach for damage identification and crack mode classification in reinforced concrete structures. Journal of Intelligent Material Systems and Structures 24(14): 1722-1735. 
Farrar CR, Doebling SW and Nix DA (2001) Vibration-based structural damage identification. Philosophical Transactions of the Royal Society of London A: Mathematical, Physical and Engineering Sciences 359(1778): 131-149.

Farrar CR and Worden K (2007) An introduction to structural health monitoring. Philosophical Transactions of the Royal Society A: Mathematical, Physical and Engineering Sciences 365(1851): 303-315.

Fassois SD and Sakellariou JS (2007) Time-series methods for fault detection and identification in vibrating structures. Philosophical Transactions of the Royal Society A: Mathematical, Physical and Engineering Sciences 365(1851): 411-448.

Fawcett T (2006) An introduction to ROC analysis. Pattern Recognition Letters 27(8): 861-874.

Figueiredo E, Figueiras J, Park G, Farrar CR and Worden $\mathrm{K}$ (2011) Influence of the autoregressive model order on damage detection. Computer-Aided Civil and Infrastructure Engineering 26(3): 225-238.

Ghoshal A, Sundaresan MJ, Schulz MJ and Pai PF (2000) Structural health monitoring techniques for wind turbine blades. Journal of Wind Engineering and Industrial Aerodynamics 85(3): 309324.

Gopalakrishnan S, Ruzzene M and Hanagud S (2011) Computational Techniques for Structural Health Monitoring. London, UK: Springer.

Hernandez-Garcia MR and Masri SF (2014) Application of statistical monitoring using latent-variable techniques for detection of faults in sensor networks. Journal of Intelligent Material Systems and Structures 25(2): 121-136.

Jain AK, Duin RPW and Mao J (2000) Statistical pattern recognition: A review. IEEE Transactions on Pattern Analysis and Machine Intelligence 22(1): 4-37.

Jensen FM, Sørensen JD, Nielsen PH, Berring P and Flores S (2011) Failures in trailing edge bondlines of wind turbine blades. In: The 32nd Ris $\phi$ International Symposium on Materials Science, volume 32. Forskningscenter Ris $\varnothing$ Materialeforskning, pp. 319-327.

Jolliffe IT (2002) Principal Component Analysis. 2nd edition. New York, USA: Springer.

Kay SM (1988) Modern Spectral Estimation: Theory and Application. Upper Saddle River, USA: Prentice-Hall.

Kostka P, Holeczek K, Filippatos A, Langkamp A and Hufenbach W (2013) In situ integrity assessment of a smart structure based on the local material damping. Journal of Intelligent Material Systems and Structures 24(3): 299-309.

Kramer O, Ciaurri DE and Koziel S (2011) Derivative-free optimization. Computational Optimization, Methods and Algorithms. Berlin, Germany: Springer, pp. 61-83.

Krzanowski WJ and Hand DJ (2009) ROC Curves for Continuous Data. Boca Raton, USA: Taylor and Francis.

Kullaa J (2003) Damage detection of the Z24 bridge using control charts. Mechanical Systems and Signal Processing 17(1): 163170.

Kumar PR, Oshima T, Yamazaki T, Mikami S and Miyamouri Y (2012) Detection and localization of small damages in a real bridge by local excitation using piezoelectric actuators. Journal of Civil Structural Health Monitoring 2(2): 97-108.

Lee SG, Yun GJ and Shang S (2014) Reference-free damage detection for truss bridge structures by continuous relative wavelet entropy method. Structural Health Monitoring 13(3): 307-320.

Li YY (2010) Hypersensitivity of strain-based indicators for structural damage identification: A review. Mechanical Systems and Signal Processing 24(3): 653-664.

Liu Y and Chattopadhyay A (2013) Low-velocity impact damage monitoring of a sandwich composite wing. Journal of Intelligent Material Systems and Structures 24(17): 20742083.

Lu KC, Loh CH, Yang YS, Lynch JP and Law KH (2008) Realtime structural damage detection using wireless sensing and monitoring system. Smart Structures and Systems 4(6): 759777.

Manson G and Barthorpe RJ (2010) Advanced feature selection for simplified pattern recognition within the damage identification framework. Shock and Vibration 17(4-5): 589-599.

Meo M (2014) Sensor Technologies for Civil Infrastructures, volume 55, chapter Acoustic emission sensors for assessing and monitoring civil infrastructures. Sawston, UK: Woodhead, pp. 159-178.

Mohd Aris KD, Mustapha F, Salit MS and Abdul Majid DLA (2014) Condition structural index using principal component analysis for undamaged, damage and repair conditions of carbon fiber-reinforced plastic laminate. Journal of Intelligent Material Systems and Structures 25(5): 575-584.

Mujica LE, Ruiz M, Pozo F, Rodellar J and Gemes A (2014) A structural damage detection indicator based on principal component analysis and statistical hypothesis testing. Smart Materials and Structures 23(2): 1-12.

Nair KK and Kiremidjian AS (2012) A wavelet-based damage diagnosis algorithm using principal component analysis. Structural Control and Health Monitoring 19(8): 672-685.

Nair KK, Kiremidjian AS and Law KH (2006) Time series-based damage detection and localization algorithm with application to the ASCE benchmark structure. Journal of Sound and Vibration 291(1-2): 349-368.

Papatheou E, Manson G, Barthorpe RJ and Worden K (2010) The use of pseudo-faults for novelty detection in SHM. Journal of Sound and Vibration 329(12): 2349-2366.

Park C, Tang J and Ding Y (2010) Aggressive data reduction for damage detection in structural health monitoring. Structural Health Monitoring 9(1): 59-74.

Prabhu S and Atamturktur S (2013) Feature assimilation for vibration based damage detection. Journal of Testing and Evaluation 41(1): 1-11.

Quenouille MH (1949) Approximate tests of correlation in timeseries. Journal of the Royal Statistical Society. Series B (Methodological) 11(1): pp. 68-84.

Rencher AC (1998) Multivariate Statistical Inference and Applications. New York, USA: Wiley.

Reynders E (2012) System identification methods for (operational) modal analysis: Review and comparison. Archives of Computational Methods in Engineering 19(1): 51-124.

Reynders E, Houbrechts J and de Roeck G (2012) Fully automated (operational) modal analysis. Mechanical Systems and Signal Processing 29: 228-250.

Rizos DD, Fassois SD, Marioli-Riga ZP and Karanika AN (2008) Vibration-based skin damage statistical detection and restoration assessment in a stiffened aircraft panel. Mechanical 
Systems and Signal Processing 22(2): 315-337.

Singhal A (2001) Modern information retrieval: A brief overview. Bulletin of the IEEE Computer Society Technical Committee on Data Engineering 24(4): 35-42.

Söderström T and Stoica P (1989) System Identification. Hemel Hempstead, UK: Prentice-Hall.

Sohn H, Czarnecki JA and Farrar CR (2000) Structural health monitoring using statistical process control. Journal of Structural Engineering 126(11): 1356-1363.

Sohn H, Farrar CR, Hemez FM, Shunk DD, Stinemates DW, Nadler BR and Czarnecki JA (2004) A review of structural health monitoring literature: 1996-2001. Technical Report LA-13976MS, Los Alamos National Laboratory, Los Alamos, USA.

Tippmann JD, Zhu X and Lanza Di Scalea F (2015) Application of damage detection methods using passive reconstruction of impulse response functions. Philosophical Transactions of the Royal Society A: Mathematical, Physical and Engineering Sciences 373(2035): 1-16.

Torres-Arredondo MA, Buethe I, Tibaduiza DA, Rodellar J and Fritzen CP (2013) Damage detection and classification in pipework using acousto-ultrasonics and non-linear data-driven modelling. Journal of Civil Structural Health Monitoring 3(4): 297-306.

Torres-Arredondo MA, Sierra Prez J, Tibaduiza DA, McGugan M, Rodellar J and Fritzen CP (2015) Signal-based nonlinear modelling for damage assessment under variable temperature conditions by means of acousto-ultrasonics. Structural Control and Health Monitoring 22(8): 11031118.

Trendafilova I, Palazzetti R and Zucchelli A (2015) Damage assessment based on general signal correlation. Application for delamination diagnosis in composite structures. European Journal of Mechanics, A/Solids 49: 197-204.

Wang Z and Ong KCG (2009) Structural damage detection using autoregressive-model-incorporating multivariate exponentially weighted moving average control chart. Engineering Structures 31(5): 1265-1275.

Worden K, Manson G, Hilson G and Pierce SG (2008) Genetic optimisation of a neural damage locator. Journal of Sound and Vibration 309(35): 529-544.

Yan AM, Kerschen G, De Boe P and Golinval JC (2005) Structural damage diagnosis under varying environmental conditions Part I: A linear analysis. Mechanical Systems and Signal Processing 19(4): 847-864.

Yang Z, Yu Z and Sun H (2007) On the cross correlation function amplitude vector and its application to structural damage detection. Mechanical Systems and Signal Processing 21(7): 2918-2932.

Yang ZC, Wang L, Wang H, Ding Y and Dang X (2009) Damage detection in composite structures using vibration response under stochastic excitation. Journal of Sound and Vibration 325(4-5): 755-768.

Ying Y, Garrett JH, Oppenheim IJ, Soibelman L, Harley JB, Shi J and Jin Y (2013) Toward data-driven structural health monitoring: Application of machine learning and signal processing to damage detection. Journal of Computing in Civil Engineering 27(6): 667-680.

Zhang M and Schmidt R (2014) Sensitivity analysis of an autocorrelation-function-based damage index and its application in structural damage detection. Journal of Sound and Vibration 333(26): 7352-7363.
Zimek A, Schubert E and Kriegel HP (2012) A survey on unsupervised outlier detection in high-dimensional numerical data. Statistical Analysis and Data Mining 5(5): 363-387.

Zugasti E, Mujica LE, Anduaga J and Martinez F (2013) Feature selection - extraction methods based on PCA and mutual information to improve damage detection problem in offshore wind turbines. Key Engineering Materials 569-570: 620-627. 